TIPA. Travaux interdisciplinaires sur la parole et le langage

36 | 2020

Comment le corps coconstruit les discours et le sens

\title{
De la transmission d'informations cliniques au partage de savoir lors de relèves infirmières
}

From the transmission of clinical information to the sharing of knowledge during nursing handovers

Isabel Colón de Carvajal, Louis Maritaud, Benoit Chalancon et Justine Lascar

\section{OpenEdition}

Édition électronique

URL : http://journals.openedition.org/tipa/4032

DOI : 10.4000/tipa.4032

ISSN : 2264-7082

Éditeur

Laboratoire Parole et Langage

Référence électronique

Isabel Colón de Carvajal, Louis Maritaud, Benoit Chalancon et Justine Lascar, « De la transmission d'informations cliniques au partage de savoir lors de relèves infirmières », TIPA. Travaux interdisciplinaires sur la parole et le langage [En ligne], 36 | 2020, mis en ligne le 01 juin 2020, consulté le 26 janvier 2021. URL : http://journals.openedition.org/tipa/4032 ; DOI : https://doi.org/10.4000/tipa. 4032

Ce document a été généré automatiquement le 26 janvier 2021.

a revue TIPA. Travaux interdisciplinaires sur la parole et le langage est mise à disposition selon les termes de la licence Creative Commons Attribution - Pas d'Utilisation Commerciale - Pas de Modification 4.0 International. 


\section{De la transmission d'informations cliniques au partage de savoir lors de relèves infirmières}

From the transmission of clinical information to the sharing of knowledge during nursing handovers

Isabel Colón de Carvajal, Louis Maritaud, Benoit Chalancon et Justine Lascar

\section{Introduction}

1 La relève infirmière est un lieu d'échanges riche en discours et en pratique, dont le principal objectif est de transmettre et partager à plusieurs des informations actualisées sur les différents patients du service concerné. Pour autant, comme l'ont expliqué Grosjean \& Lacoste (1999: 5), « le rôle de l'interaction ce n'est pas seulement de transmettre à sens unique, c'est répondre aux questions et aux sollicitations des autres et intégrer leurs réactions, c'est construire un discours à plusieurs ». Ainsi, les participants aux relèves "ne se contentent pas d'énoncer les faits récents, mais donnent l'occasion de s'interroger sur eux, de chercher à les interpréter, à compléter des savoirs, d'établir des jugements, de reconstituer des événements et des problèmes, d'aider le partenaire à y voir clair » (Grosjean \& Lacoste, 1999 : 112).

Dans ce contexte de relèves infirmières, nous menons une étude observationnelle et descriptive en collaboration entre l'hôpital le Vinatier et le laboratoire ICAR (CNRS, Université Lyon 2, ENS de Lyon) dans un service de soins psychiatriques. Il s'agit du projet CIPSY (Compétence d'Interaction des Infirmiers autour du dossier patient en PSYchiatrie) dont l'objectif principal est de déterminer le répertoire langagier des deux formats de production (orale et écrite) lors des relèves infirmières et des saisies d'observations écrites, au moyen d'analyses qualitatives et quantitatives. En effet, le contexte actuel de la santé mentale augmente de plus en plus le nombre d'intervenants autour du patient, le développement des spécialisations en psychiatrie multipliant les 
acteurs à son contact. Ce paysage où les collaborateurs se diversifient au service du soin met également en avant toute l'importance d'une communication interprofessionnelle performante. Cette complexification favorable aux soins donne une dimension toute particulière aux transmissions orales et écrites dans les équipes de soignants. Les données de cette étude ont été recueillies lors de captations audio et vidéo dans le contexte singulier d'un hôpital psychiatrique. L'accès à ce terrain ne fut possible qu'avec la participation des équipes soignantes. La mise en avant des techniques interactionnelles mobilisées dans ces séquences met en valeur leurs compétences spécifiques. Aussi, les enjeux de la relève pour le soin sont illustrés à travers ces données et démontrent également toute l'importance de favoriser la culture des relèves dans un souci d'amélioration de la qualité des soins.

3 L'organisation habituelle d'une relève met en valeur un participant principal (que nous appellerons super locuteur) qui mène le déroulement des transmissions patient par patient. D'un point de vue interactionnel, deux moments modifient l'organisation thématique de la relève pour laisser place à des séquences où les co-participants interviennent soit pour réguler la transmission en cours (ce qui relèverait d'un « faire savoir »), soit pour partager des connaissances professionnelles sur un médicament/ produit (ce qui relèverait d'un « faire apprendre »).

4 Nous reprenons cette conception du « discours didactique » des travaux de Moirand (1993) et la distinction entre « faire savoir » et «faire apprendre » proposée fait suite à l'analyse interactionnelle et multimodale des données. Ainsi, nous montrerons d'une part, comment les co-participants "font savoir» au super locuteur qu'il existe une erreur dans la transition d'un patient à l'autre. Comment un co-participant suspend-il la progression de la transition vers un autre patient, et comment fait-il savoir au super locuteur qu'il n'a pas terminé de prendre ses notes sur sa feuille de relève ? D'autre part, nous décrirons comment les participants traitent l'émergence d'échanges de connaissances partagées sur le métier. La problématique centrale de cet article sera donc de rendre compte finement de l'importance des ressources multimodales (i.e. des ressources corporelles) dans la co-construction de l'échange professionnel et plus particulièrement dans des séquences où l'on observe une distinction entre ceux qui « savent » et ceux qui « devraient savoir » ou « font savoir ».

5 Après avoir résumé les études sur les relèves infirmières dans une perspective interactionniste, nous présenterons brièvement notre ancrage théorique, puis le corpus, et la méthodologie de terrain appliquée. Nous expliquerons ensuite l'organisation des relèves telles qu'elles se déroulent dans le service psychiatrique enregistré et nous exposerons en quoi ces relèves ont selon nous une portée, une intention didactique dans l'échange. Nous proposerons une partie analytique en deux points : premièrement, l'analyse de deux extraits correspondant à des séquences de "faire savoir " lors d'une transition multimodale réparée d'un patient à l'autre; deuxièmement, l'analyse de deux autres extraits relatifs à des moments de "faire apprendre " pendant le partage de connaissance professionnelle. Nous terminerons enfin par une mise en perspective des résultats présentés. 


\section{Analyse des relèves infirmières dans une perspective interactionniste}

6 Les conversations en contexte de relève infirmière ont fait l'objet de différentes études depuis plusieurs années, selon différents contextes (service de chirurgie, de pédiatrie, d'urgence, de psychiatrie, etc.). En France, on peut citer notamment les travaux centraux de Grosjean (1997, 2004) et Grosjean \& Lacoste (1999) dans lesquels les autrices se sont particulièrement intéressées à décrire les pratiques des professionnels dans ce contexte. L'édition de 1999 constitue un ouvrage de référence sur le sujet des relèves. À l'international, les chercheurs ont étudié la coopération entre infirmiers et médecins (Lanza et al., 2004) ou le discours rapporté dans des récits pendant la relève (Bangerter et al., 2011). Plus récemment, Abraham et al. (2015) ont mené une analyse conversationnelle et séquentielle de la structure et des contenus d'une relève ; LeBaron et al. (2016) ont conduit une étude ethnométhodologique des routines conversationnelles; enfin Mori et al. (2017) ont montré comment les infirmières travaillant dans un service gériatrique japonais rapportent, confirment et reconstruisent les informations lors des relèves. Ainsi, dans les travaux cités, nous avons remarqué qu'aucun ne mettait en lumière une analyse interactionnelle et multimodale fine de séquence pour « compléter des savoirs » ou « aider le partenaire à y voir clair», ces échanges ne constituant pas normalement la part principale de la relève.

7 Plus largement, notre étude sur les relèves infirmières s'inscrit dans le champ de l'analyse conversationnelle qui est une discipline développée par Sacks à la fin des années 60, puis en étroite collaboration avec Schegloff et Jefferson, pour étudier l'organisation sociale de l'action in situ (Sacks et al., 1974; Sacks, 1992). Elle s'inspire de l'ethnométhodologie de Garfinkel (Garfinkel, 1967). Initialement axée sur l'étude de l'interaction parlée (à partir de données audio), un travail de plus en plus important est mené (à partir de données audio et vidéo) et prend en compte - au-delà de la parole - le geste, le regard, la posture et d'autres moyens de communication au cours des conversations.

8 Lorsqu'on s'intéresse à la relation entre geste et parole dans l'interaction humaine, il est souvent d'usage de s'appuyer sur des classifications des différents types de gestes produits par les locuteurs, comme le rappelle Kendon (2004, p. 103). Il propose d'ailleurs une synthèse éclairante de trois classifications gestuelles majeures dans les travaux sur les gestes (ibid., p. 103) :

1. les " gestes considérés » où l'on distingue les gestes réalisés avec les mains et les bras en opposition aux autres mouvements du corps (tête, yeux, etc.) en renvoyant ici aux travaux de référence d'Austin (1806), d'Ekman \& Friesen (1967);

2. le lien entre les gestes et le discours qui peut être nuancé entre l'expression du corps et le discours. Une distinction est donc possible entre les liens unissant une culture et une correspondance gestuelle/discursive (Efron, 1941) et la relation entre les processus de pensée et l'expression linguistique (McNeill, 1992, 2000);

3. les fonctions sémiotiques des gestes où l'on distingue par exemple les gestes déictiques des gestes démonstratifs ; les gestes imitatifs des gestes expressifs (Cosnier \& Vaysse, 1997).

9 Cependant, les différentes typologies présentent des difficultés inhérentes à la classification, telles que la délimitation efficace des gestes et l'exclusion mutuelle de 
différentes catégories qui ne correspondent pas aux gestes trouvés en contexte (Kendon, 2004 : 104).

10 Les conversationnalistes qui étudient l'interaction au-delà du discours définissent de plus en plus leur travail en termes de multimodalité. En effet, l'action humaine est fondamentalement multimodale (Mondada, 2014 : 139). Nous mobilisons donc plus facilement la notion de "ressource » (i.e. la ressource multimodale) en la décrivant sous trois considérations :

«i) to treat linguistic and embodied resources in principle in the same way, without prioritizing a priori one type of resource over other ones; ii) to identify not only conventionalized sets of resources, as grammar and some types of gesture, but also situatedly occasioned and assembled resources; iii) to study how resources are combined together in various configurations, depending on the activity, its ecology and its material and cultural constraints. » (ibid.).

11 Par conséquent, lorsque nous analysons la relation entre les gestes et la parole, il est également important de considérer comment les participants synchronisent les différentes ressources disponibles en fonction de l'activité en cours :

«In some human activities, language plays a crucial role, while in other activities other resources are privileged: thus, the prioritization of one resource over the other is not a matter that can be decided in a principled way, but an empirical issue that depends on the type of situated activity and on the way in which participants format it. » (ibid.).

Dans notre article, nous considérons ainsi les gestes comme une ressource, tout comme le langage, pour interagir et effectuer des actions quotidiennes. Aussi, nous considérons les gestes comme des ressources potentiellement co-interprétées par les coparticipants pour assurer la compréhension mutuelle de l'échange :

«Gesture as meaningful action is accomplished not by a speaker's hand alone, but instead through the relevant juxtaposition of a range of different kinds of semiotic materials which mutually elaborate each other. " (Goodwin, $2000: 84$ ).

13 Pour explorer comment l'interaction multimodale se déroule moment par moment, nous utilisons une transcription fine et une analyse de petites séquences d'enregistrements vidéo. Nous démontrons à partir de ces transcriptions : a) comment les individus coordonnent leurs actions et anticipent les actions des autres dans le travail collaboratif ; b) comment les personnes négocient les rôles et se positionnent par rapport aux autres ; et c) comment les participants utilisent toutes les parties de leur corps en parallèle ou en l'absence de parole. Un concept clé dans ce travail et plus généralement en analyse conversationnelle est la notion de séquentialité, c'est-à-dire l'ordre temporel de l'action comme un moyen d'atteindre une compréhension mutuelle.

\section{Corpus et méthodologie de terrain}

14 Afin de répondre aux interrogations de l'étude CIPSY, l'équipe de recherche a fait le choix de créer un corpus de données in situ. La captation des données a été réalisée par des enregistrements audio et vidéo à l'occasion des relèves de postes inter équipes. Le matériel mis en place était composé de trois caméras et cinq micros. À des fins de discrétion, nous avons utilisé des "pocket cam » à trépied adaptatif. La prise de son a été assurée par des micro HF, ainsi qu'un micro d'ambiance, posés sur table. L'équipe de 
recherche venait simplement allumer et éteindre l'enregistrement, limitant ainsi le contact avec l'équipe de soin, avec le plus de discrétion possible.

Le choix du lieu de tournage a, en premier lieu, suivi tout un parcours d'autorisations institutionnelles au sein de l'hôpital. L'étude, perçue comme une opportunité pour l'encadrement des équipes, a été proposée sur le pôle depuis lequel elle s'est développée. L'une des équipes ainsi sollicitées s'est montrée plus particulièrement favorable à l'enregistrement des données. Après la présentation de l'étude aux équipes de jour et de nuit et le recueil des autorisations de droit à l'image, la programmation des jours de tournage a pu se décider.

Le choix de l'équipe investiguée s'est porté vers le tournage des lundis uniquement. Le lundi en tant que premier jour de la semaine est, pour un service de psychiatrie adulte, d'une richesse particulière, puisqu'abondant d'évènements cliniques survenus le weekend. La particularité de ce jour tient également au déroulement du staff hebdomadaire ${ }^{1}$. Ce corpus audiovisuel original, d'une durée totale de $14 \mathrm{~h} 30$, met en images 38 professionnels de santé, et concerne 40 patients mis à l'ordre du jour de ces relèves (sur cinq lundis seulement).

La phase de captation, une fois réalisée, a donné lieu à une seconde phase de traitement des données (synchronisations multi-vues, voir illustrations à la section suivante) et à une partie de leurs transcriptions et codages dans le logiciel ELAN ${ }^{2}$ (Wittenburg et al., 2006).

18 En parallèle de ces actions, les données écrites correspondant aux cinq lundis enregistrés ont été collectées, puis transcrites dans le logiciel CLAN ${ }^{3}$ (MacWhinney, 2000). Elles sont analysées au moyen d'une grille de codage commune à celle des données orales.

La méthodologie de travail de terrain, de recueil de corpus et d'analyse dans laquelle nous nous inscrivons est celle développée en linguistique interactionnelle et en analyse conversationnelle. Cette méthodologie vise à capturer des données audio et vidéo afin de rendre disponibles, et donc analysables, les détails linguistiques, multimodaux et situationnels (regards, gestes, mouvements, actions, objets, cadre physique) pertinents pour l'interaction enregistrée (Groupe ICOR, 2006). Elle permet aussi d'observer précisément l'évolution dans le temps et dans l'espace de l'activité, les interactions sociales qui se co-construisent entre les participants.

Les premiers résultats présentés dans cet article sont construits sur l'analyse multimodale de six patients, représentant 3 heures 42 minutes de tournage (soit 25,51 $\%$ du corpus total). Le choix de ces six patients s'est porté sur leur modalité de séjour hospitalier.

CESEL : il a débuté son séjour durant l'étude, et a été placé dans l'espace de soin psychiatrique intensif.

DUBYV : le patient est sorti, puis a été ré-admis.

LEBBA : le patient a terminé son séjour durant le tournage.

LEGCO : le patient a débuté son séjour hospitalier. 
TOUJE et DELEM : ils ne sont ni rentrés, ni sortis durant la phase de captation, étant ainsi au cœur de leur hospitalisation.

La transcription de cet échantillon a nécessité $222 \mathrm{~h}$ de travail, et $240 \mathrm{~h}$ pour le codage. Les réflexions présentées ici s'appuient donc sur une analyse qualitative particulièrement détaillée, mais ne concernent qu'un faible échantillon de patients (seulement $15 \%$ du corpus).

\section{Les relèves infirmières et leur intention didactique}

Dans nos données, le rythme de travail des infirmiers est assez similaire à un roulement en « trois-huit ». Les roulements s'enchaînent ainsi : $6 \mathrm{~h} 30>14 \mathrm{~h} 30$ puis $14 \mathrm{~h}>22 \mathrm{~h}$ puis $21 \mathrm{~h} 45>6 \mathrm{~h} 45$. Ainsi, le chevauchement des équipes est d'une durée de quinze à trente minutes, réparties sur trois relèves : nuit/jour, matin/après-midi, et après-midi/ nuit, auxquelles s'ajoute la réunion médicale de la semaine appelée aussi «staff hebdomadaire ".

- Relève nuit/jour : 6 h 30 - 6 h 45

Cette première relève de la journée se déroule dans le bureau infirmier, les recommandations de l'institution invitent l'aide-soignant de nuit à faire le tour physique de l'unité avec un soignant de jour. Tous deux ne peuvent donc pas assister à la relève orale. Restent alors dans le bureau l'infirmier de nuit et souvent deux infirmiers du matin. C'est une relève assise, en équipe restreinte. Cette relève a une durée de dix-sept minutes en moyenne.

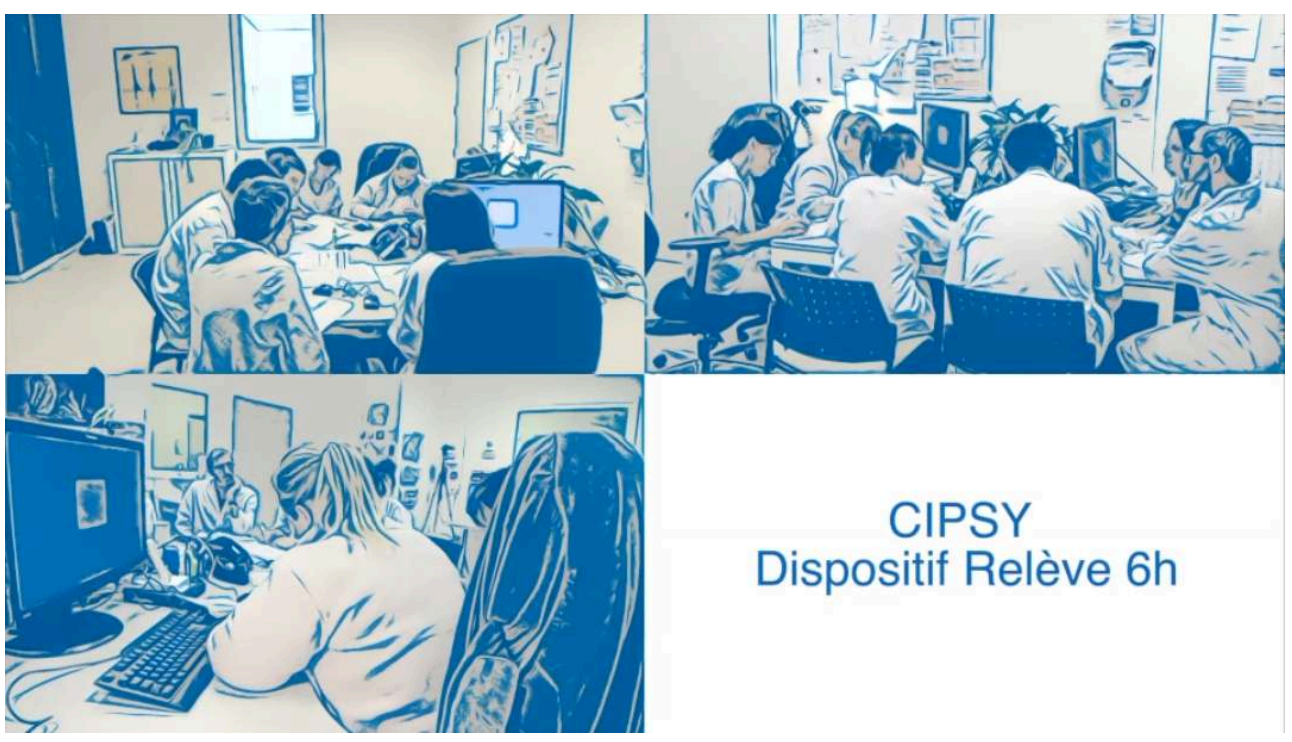

(c) Laboratoire ICAR (UMR5191)

- Relève matin/après-midi : $14 \mathrm{~h}$ - $14 \mathrm{~h} 30$

Elle se déroule en salle de réunion pour une raison de places disponibles (le bureau infirmier serait trop petit), mais également pour des raisons techniques, puisque cette pièce est équipée d'un support de vidéo-projection. Les ASH (Agents de Service Hospitalier) sont également présents pour créer un temps de réunion essentiellement entre personnels soignants non médicaux. Les durées de cette relève dans notre étude 
varient entre trente minutes et une heure. C'est la réunion la plus sensible aux variations de durée.

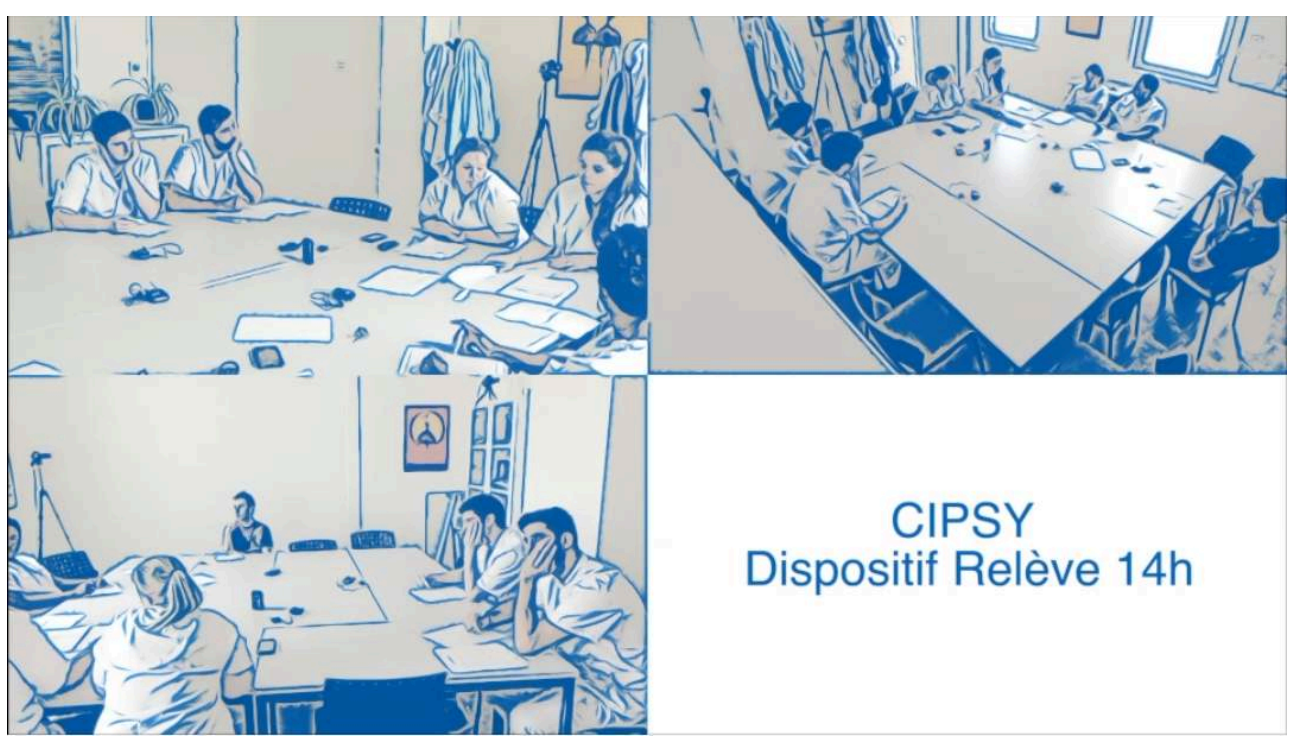

(c) Laboratoire ICAR (UMR5191)

- Relève après-midi/nuit : 21 h 45 - 22 h

Ce dernier temps de transmission est assez identique à celui du matin, les mêmes personnes sont présentes et le même rituel de séparation se déroule entre ceux qui ont accès à la relève orale et ceux qui vont faire le tour du service. Elles durent de vingt à quarante minutes.

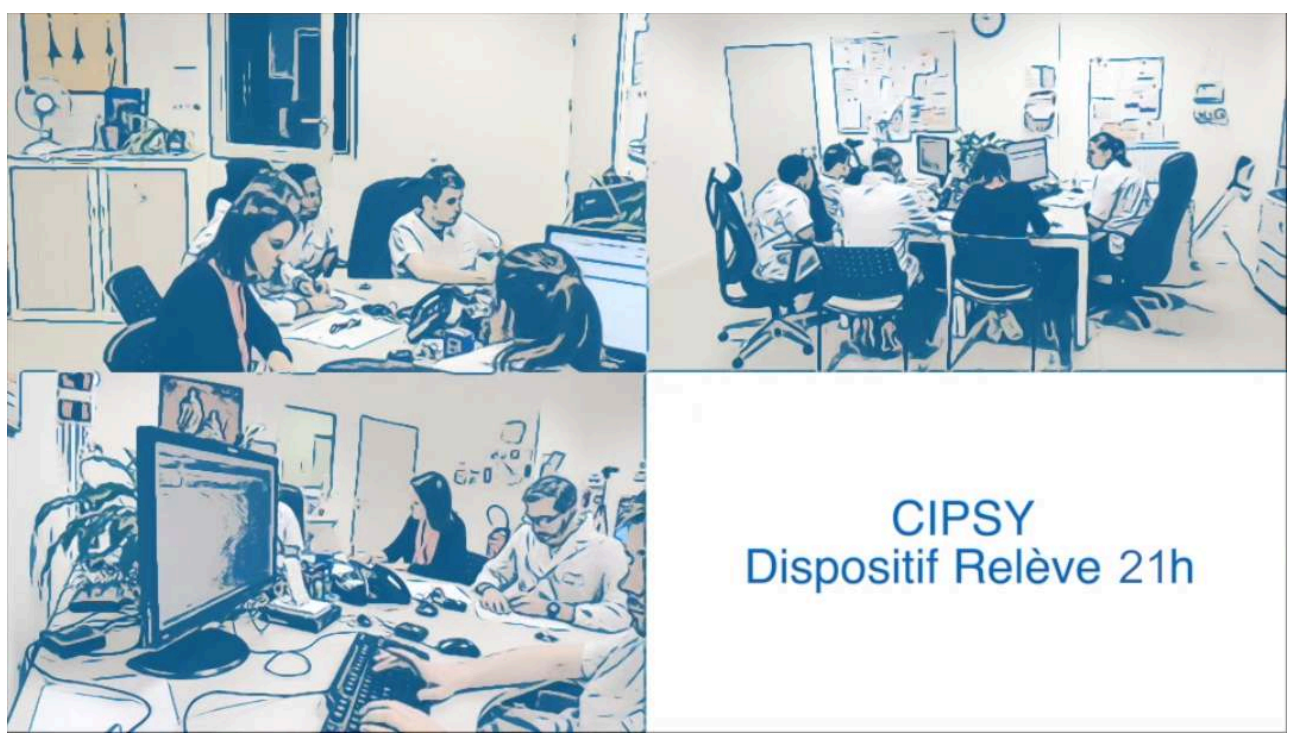

(c) Laboratoire ICAR (UMR5191)

- Staff hebdomadaire : $9 \mathrm{~h}-\mathbf{1 0} \mathrm{h} \mathbf{3 0}$

Le «staff hebdomadaire " aussi intitulé " réunion médicale de semaine » se déroule en salle de réunion et utilise parfois l'outil informatique et la vidéo projection. L'ensemble des patients présents dans le service à la date de la relève sont à l'ordre du jour. Chaque présentation de patient reprend l'actualité significative du week-end, donnant lieu à d'éventuels ajustements thérapeutiques. Il est également question de leur projet de soin sur les jours à venir. La composition pluridisciplinaire de l'assemblée fait de ce 
temps de réunion un moment particulièrement riche d'échanges et favorable à la coréflexion. $\mathrm{Y}$ sont présents les infirmiers et aides-soignants du matin, un ou deux médecins psychiatres, la psychologue, le cadre de santé, l'ergothérapeute, l'assistante sociale, et les étudiants (infirmiers et externes). Cette réunion n'est donc pas uniquement destinée aux soignants non médicaux mais s'ouvre au contraire à une pluridisciplinarité de l'ensemble des acteurs du service.

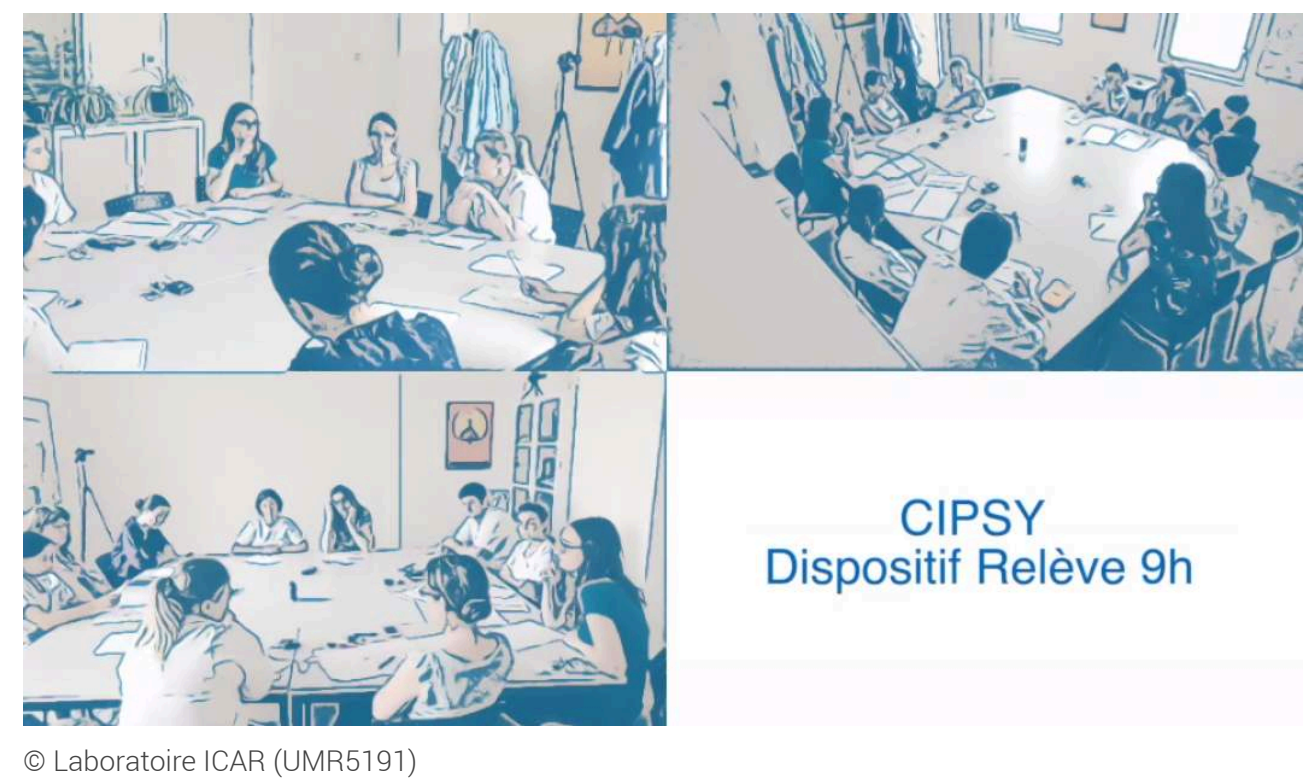

Le principal support de notes pour l'ensemble des membres de l'équipe est ce que l'on appelle la relève papier. Il s'agit d'un document interne qui regroupe sous forme de tableau l'ensemble des patients du jour. Ce tableau, en deux colonnes, précise également le numéro de chambre des patients, leur secteur géographique d'appartenance, et le médecin psychiatre référent. La seconde colonne est vide pour laisser place à la saisie manuscrite et individuelle de notes par le détenteur de la feuille. Nous y retrouvons des éléments tels que des prises de rendez-vous médicaux, des éléments de description clinique, voire des points sur le traitement. On retrouve également en bas de page des numéros d'urgences tels que celui du SAMU, celui de la sécurité incendie ou de l'interne de garde. Chaque numéro étant propre à l'hôpital, on peut y remarquer la présence d'un indicatif spécifique pour pouvoir émettre l'appel. La description de ces prises de notes fait partie d'une étape ultérieure du projet. Elles restent néanmoins une activité centrale dans le déroulement et la progression des transmissions à l'intérieur d'une relève donnée, comme nous le verrons dans l'analyse de l'extrait 1 illustrant une transition multimodale réparée.

Les relèves infirmières (hormis le staff hebdomadaire) suivent un déroulement à peu près similaire. À chaque fois, sur ces moments de transmissions, un infirmier en particulier se désigne ou est désigné pour énumérer tour à tour chaque patient, suivant un ordre établi par les numéros de chambre. Pour chaque patient mentionné, il réalise alors un bref énoncé de son actualité clinique du jour, alors que les autres participants notent (potentiellement) sur une feuille de relève ce qui est dit par l'infirmier en charge. Cette place centrale occupée par l'infirmier à l'initiative de l'énoncé du nom du patient pourrait se définir par le concept de «super locuteur». Plus qu'un rôle purement pratique, dans l'exercice infirmier, le super locuteur se distingue sur le plan 
conversationnel des autres soignants présents lors de ces relèves, comme nous allons le voir dans les prochains extraits.

Enfin, l'intention didactique de ces relèves est clairement observable par la richesse des informations transmises. Les soignants parlent bien de "transmission d'information " à propos d'un patient pendant une relève ce qui reprend l'objectif de «faire savoir/ apprendre " comme le propose Moirand (1993). Dans nos données, les discours didactiques sont produits dans le cadre d'interactions en face à face mais ne relèvent cependant pas d'interactions asymétriques dans lesquelles un expert doit adapter son discours, notamment du point de vue lexical, pour un non expert pour expliquer ou convaincre les participants à ces relèves. En effet, dans les quatre extraits analysés par la suite, les participants impliqués dans l'échange sont hiérarchiquement au même niveau et l'intention didactique de leurs discours permet soit de réparer une transition multimodale entre deux patients (extraits 1 et 2 ) : « faire savoir »; soit de faire partager des connaissances professionnelles à ses collègues (extraits 3 et 4$):$ « faire apprendre ».

\section{5. «Faire savoir » lors d'une transition multimodale réparée d'un patient à l'autre}

Dans les deux extraits à suivre, nous pouvons observer une transition multimodale réparée lors du passage d'un patient $\mathrm{A}$ à un patient $\mathrm{B}$. En effet, comme nous l'avons déjà décrit, le rôle de super locuteur implique, sur le plan interactionnel, de gérer les transitions entre chaque patient, et par conséquent d'ouvrir et de clôturer chaque séquence de description de l'actualité thérapeutique des personnes hospitalisées dans la structure.

31 La situation du premier extrait ci-dessous se déroule lors d'une relève de 14 heures, à laquelle sont donc présents uniquement des infirmiers et infirmières. Au cours de cette relève, GIL, qui occupe la place de super locuteur et qui passe donc en revue les différents patients en décrivant leur actualité clinique, va être interrompue par PAC, une autre infirmière, dans sa gestion de la machinerie des tours de parole (Sacks et al., 1978). 


\section{Extrait 1 : attends juste deux secondes}

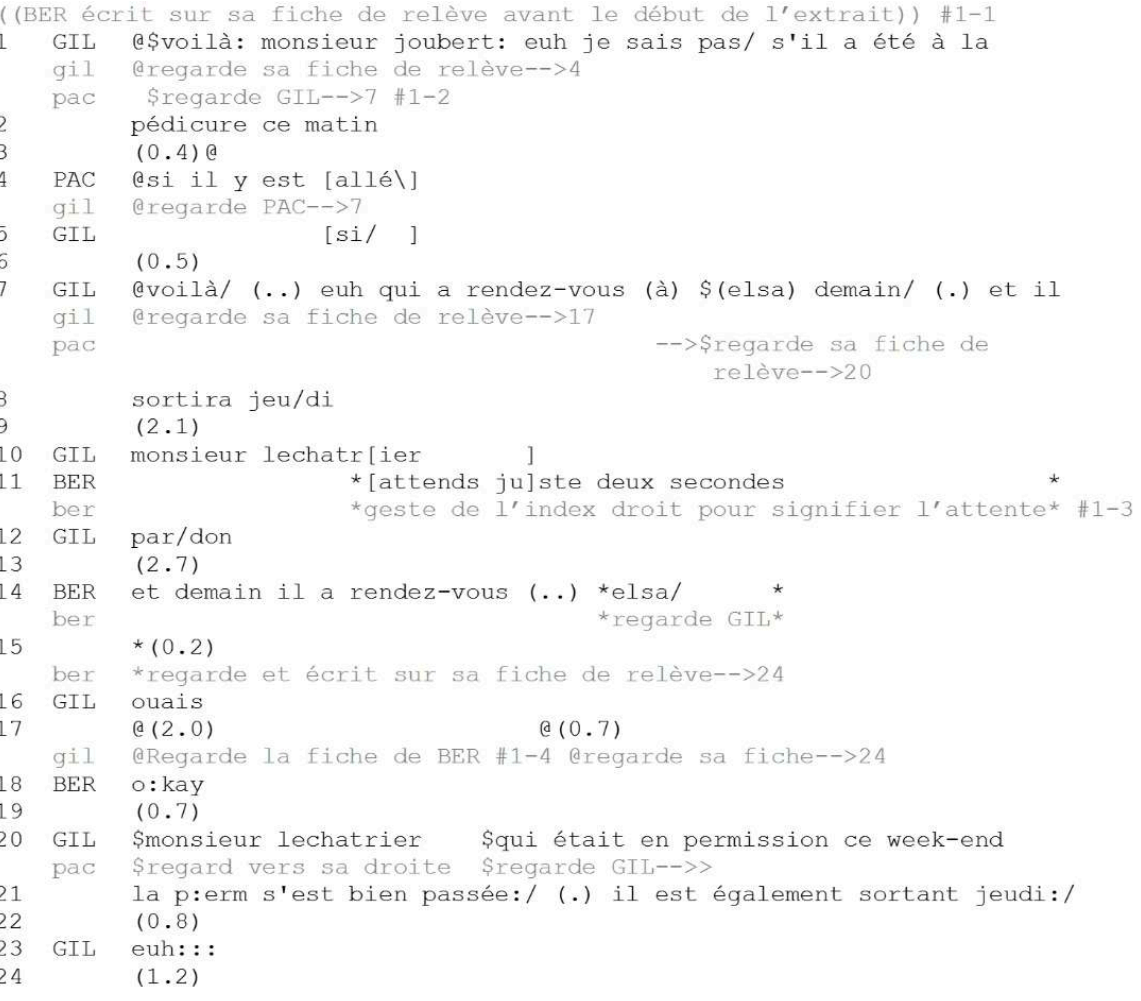

Tout d'abord, il est intéressant de noter l'activité à laquelle se livre BER au début de l'extrait : sur sa fiche de relève, elle note l'actualité clinique du patient précédemment mentionné ( $c f$. image \#1-1). Si du point de vue verbal, le rôle clé de la relève est celui du super locuteur, du point de vue multimodal, les différents participants à ces réunions de relèves sont pris dans des activités diverses, dont celle de noter ce qui est dit. Ces activités sont coordonnées, les prises de notes étant consécutives à l'énonciation du super locuteur. Cette analyse rend compte d'une difficulté de coordination des interlocuteurs, qui modifie la co-construction de l'interaction.

\#1-1

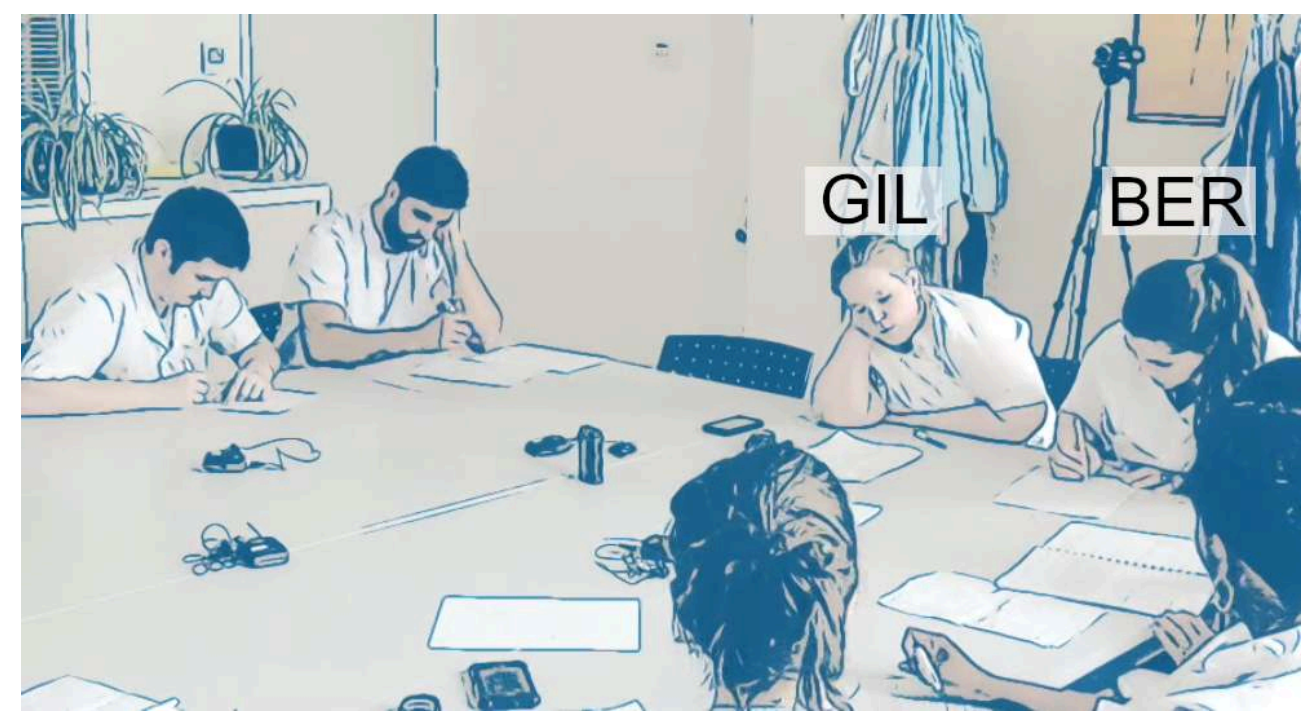

(c) Laboratoire ICAR (UMR5191)

TIPA. Travaux interdisciplinaires sur la parole et le langage, 36 | 2020 


\section{de la relève concernant un patient, sous la forme d'une subordonnée relative. Ce n'est} pas le cas dans ces premières lignes, GIL sollicitant PAC par un regard (cf. image \#1-2) pour obtenir une information, sur la pédicure effective ou non du patient en question. Suite à la paire adjacente complétée par PAC «si il y est allé » (l. 4), et la réception de l'information par GIL (1. 5), la relève concernant monsieur Joubert peut commencer, et comme on peut le constater ligne 7, la subordonnée relative est bien présente, ainsi que la reprise de " voilà ", comme observé ligne 1. Lignes 7-8, GIL endosse pleinement son rôle de super locuteur en passant en revue l'actualité clinique du patient en question, tout en lisant sa fiche de relève, son regard étant orienté vers sa feuille. Il est par ailleurs intéressant de remarquer que les transitions d'un patient à l'autre, du point de vue multimodal, se manifestent par la lecture de la fiche de relève par le super locuteur, d'une part pour pouvoir s'assurer de l'exhaustivité des informations données, et d'autre part pour pouvoir poursuivre en cherchant le patient suivant sur la feuille, et en lisant son actualité clinique.

\section{\#1-2}

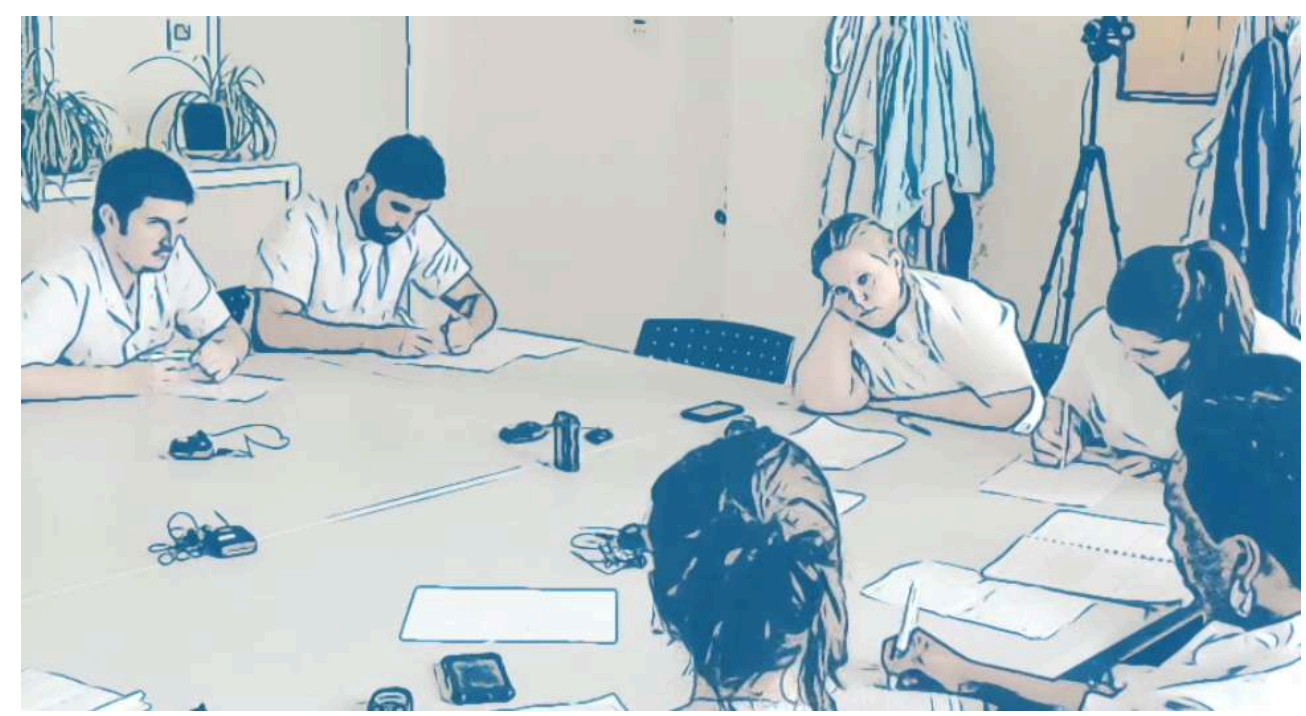

(c) Laboratoire ICAR (UMR5191)

S'ensuit une pause plutôt longue (l. 9), habituelle et représentative dans le déroulé traditionnel des transitions de patient à patient. Durant cette pause, BER continue de noter les informations, comme depuis le début de l'extrait. En dépit de cet indice multimodal (dans l'absence d'appréciation verbale de fin d'activité de la part de BER et sa gestuelle), GIL clôt la séquence concernant monsieur Joubert pour ouvrir celle concernant le patient suivant, monsieur Lechatrier. Cependant, pour manifester le besoin de temps supplémentaire, BER va produire un énoncé ligne 10 «attends juste

TIPA. Travaux interdisciplinaires sur la parole et le langage, 36 | 2020 
deux secondes ", et simultanément, lever l'index de la main droite vers GIL (cf. image \#1-3) afin d'attirer son attention, et de lui offrir un faisceau d'indices verbaux et multimodaux dans le but de se laisser le temps de noter de manière exhaustive les informations mentionnées. Ainsi, par sa gestuelle, BER initie une séquence latérale (Jefferson, 1972). Ligne 12, GIL s'excuse et prend en considération la requête de BER dans sa dimension temporelle. On pourrait considérer ce geste de BER comme résultant de l'absence de signal intra-tour (Duncan \& Fiske, 1977 ; Cosnier \& Vaysse, 1997). En effet, pour s'assurer de la bonne transmission du message, le locuteur produit parfois des gestes ou regards orientés vers ses interlocuteurs. Dans notre extrait, comme nous pouvons le remarquer dans la transcription, GIL ne lève pas les yeux de sa feuille de relève durant la pause de 2,1 secondes, et par conséquent ne peut pas remarquer que BER poursuit son activité de prise de notes. Dès lors, lever l'index lui permet de capter l'attention de GIL, et par conséquent lui signaler le problème.

\#1-3

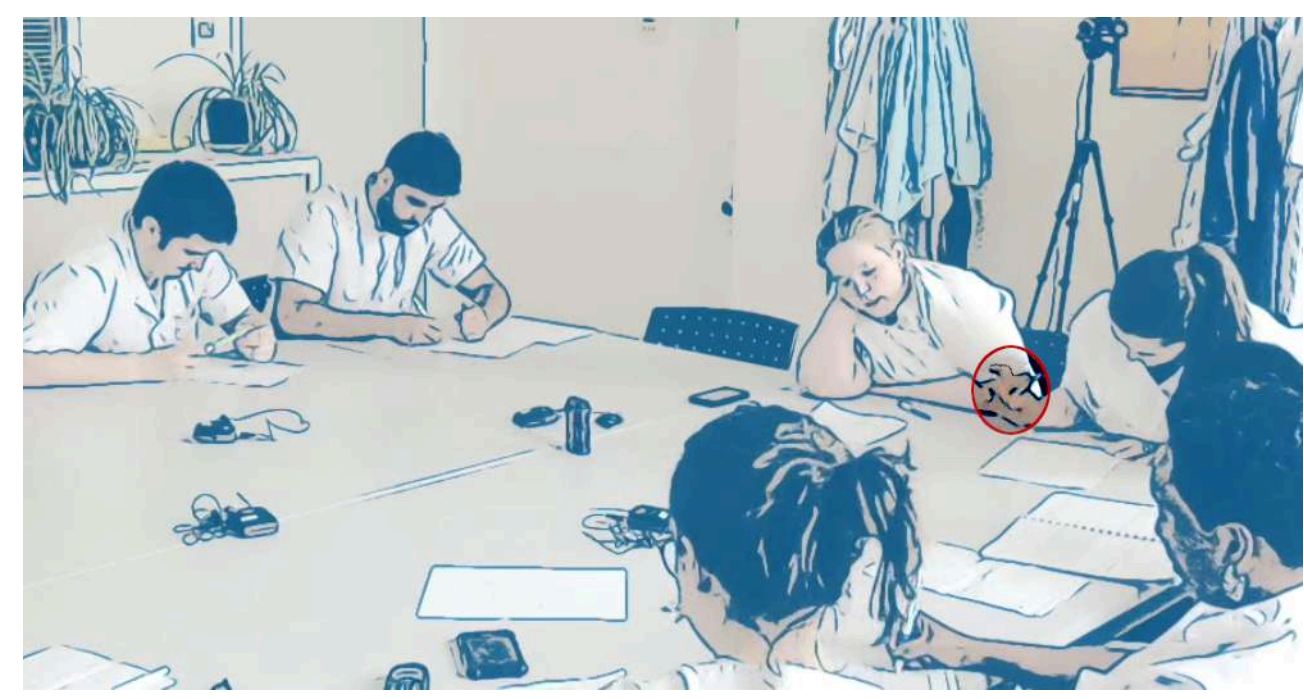

(C) Laboratoire ICAR (UMR5191)

Durant la pause de 2,7 secondes (1. 13), les soignants prennent des notes sur leur feuille de relève. Ligne 14, BER fait une répétition d'un tour précédent, sous forme de demande, adressée à GIL par le regard. Une fois cette paire adjacente (Schegloff \& Sacks, 1973) complétée par GIL (1. 17), l'activité d'écriture peut se terminer durant la pause (l. 18), pendant laquelle GIL s'assure du bon déroulé de la pratique de BER en regardant sur sa feuille de relève ( $c f$. image \#1-4). BER clôt ensuite la séquence latérale en signalant la fin de l'activité d'écriture (1. 18), par l'utilisation d'un « okay ». La relève peut alors se poursuivre avec l'actualité de monsieur Lechatrier, prochain patient sur la fiche (1. 20-24). 


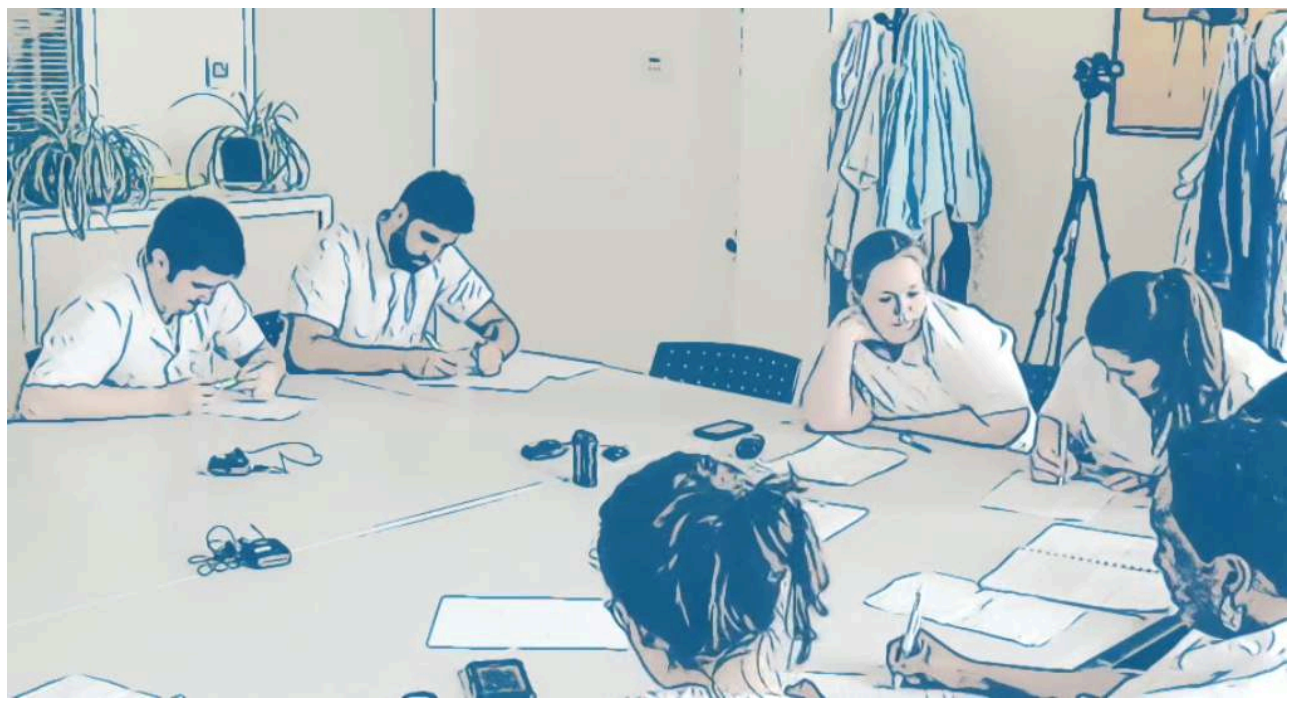

(c) Laboratoire ICAR (UMR5191)

Ce premier extrait montre donc comment l'infirmier en position de super locuteur gère la continuité de l'activité. Cependant, dans cette activité croisant plusieurs actions, dont celle de la transmission orale des informations par le super locuteur et celle de la prise de notes par les soignants, les autres participants peuvent, si nécessaire, prendre en charge une séquence latérale pour négocier avec le super locuteur la gestion du temps. Les participants coordonnent notamment cette séquence latérale d'un point de vue multimodal, à l'intérieur d'une séquence plus large concernant l'actualité clinique d'un patient. Autrement dit, « le faire savoir », qui est en train d'être opéré par le super locuteur, est alors temporisé par les réalisations multimodales (verbale et gestuelle) d'un autre soignant engagé dans son activité de prise de notes. Par cet ajustement et cette coordination multimodale entre les participants, ceux-ci rendent alors observable la portée didactique des informations transmises par le super locuteur en charge de la relève.

Dans le second extrait, ce qui pose un problème dans le déroulé de l'activité n'est pas, comme précédemment, l'aspect temporel, mais une erreur de suivi de la feuille de relève. En effet, PAC, qui est super locuteur, passe directement du patient de la chambre 11 à celui de la chambre 13, en oubliant la patiente chambre 12. Les autres participants, bien que n'endossant pas le rôle de super locuteur, et n'ayant pas pour tâche de gérer le déroulé de la réunion, vont alors s'auto-sélectionner pour négocier le bon suivi du processus, et permettre à la patiente oubliée d'avoir son actualité clinique passée en revue. Il est également important de noter que cette réunion de relève comporte plus de participants, dont le cadre de santé d'un autre service (ici en jaune). Cependant, seules trois infirmières prendront la parole au cours de cet extrait, GRI, BER et PAC. 


\section{Extrait 2 : sarrazino à la douze}

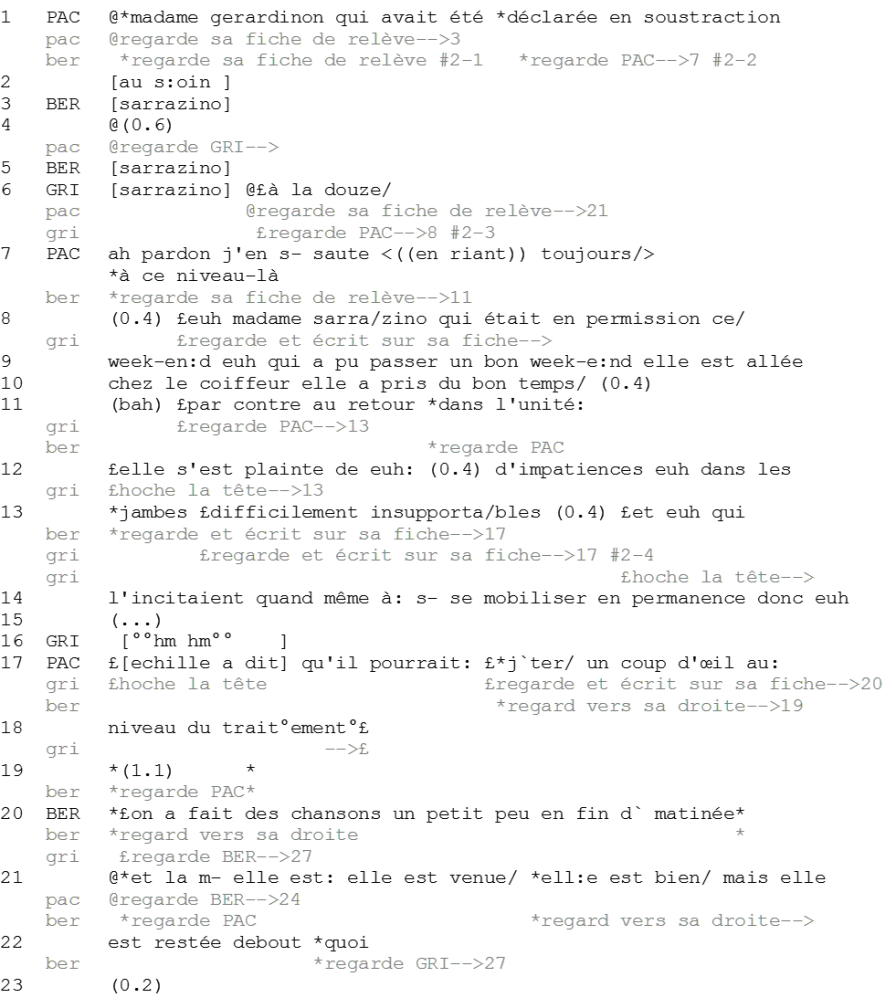

Dans cet extrait, l'ouverture de la séquence de la nouvelle patiente, madame Gérardinon, se fait en suivant la structure syntaxique habituelle : "madame Gérardinon, qui... » (l. 1). En réaction à l'ouverture de cette nouvelle séquence, BER regarde sa fiche de relève ( $c f$. image \#2-1), procède à une vérification, puis regarde PAC lorsqu'elle se rend compte de l'erreur dans le déroulé de l'activité (cf. image \#2-2).

\#2-1

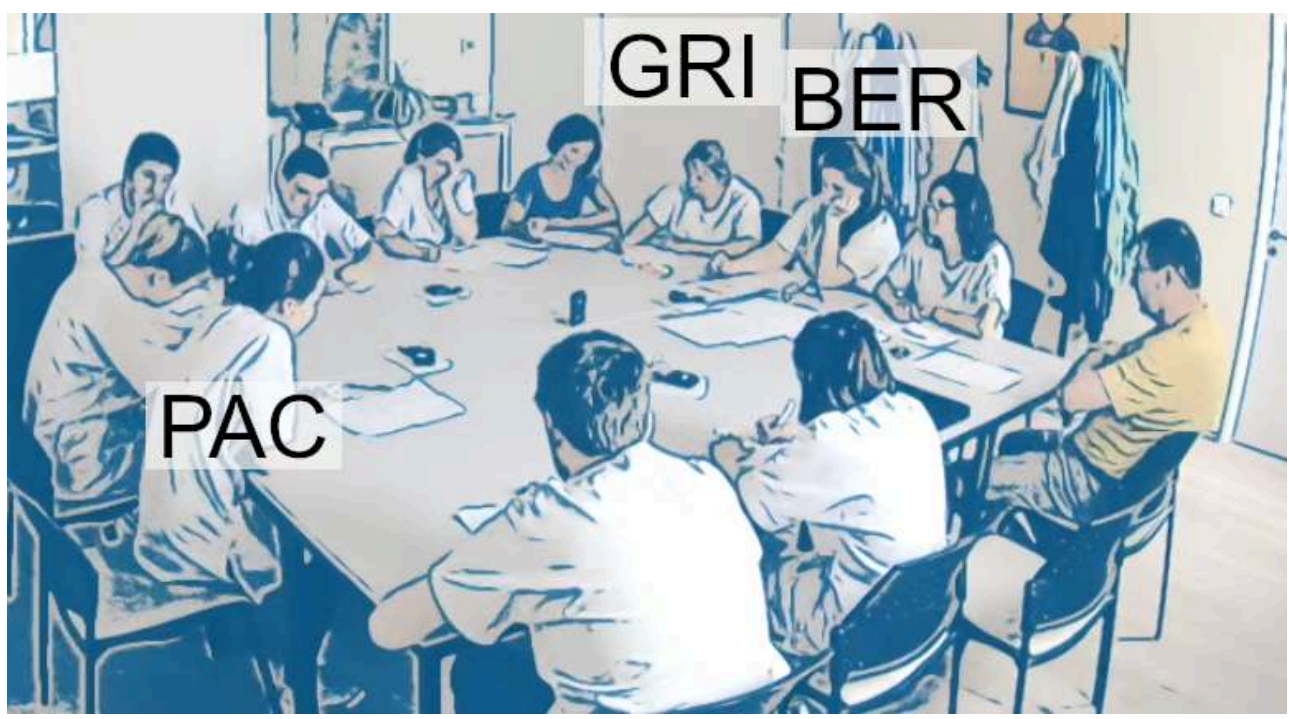

(c) Laboratoire ICAR (UMR5191) 


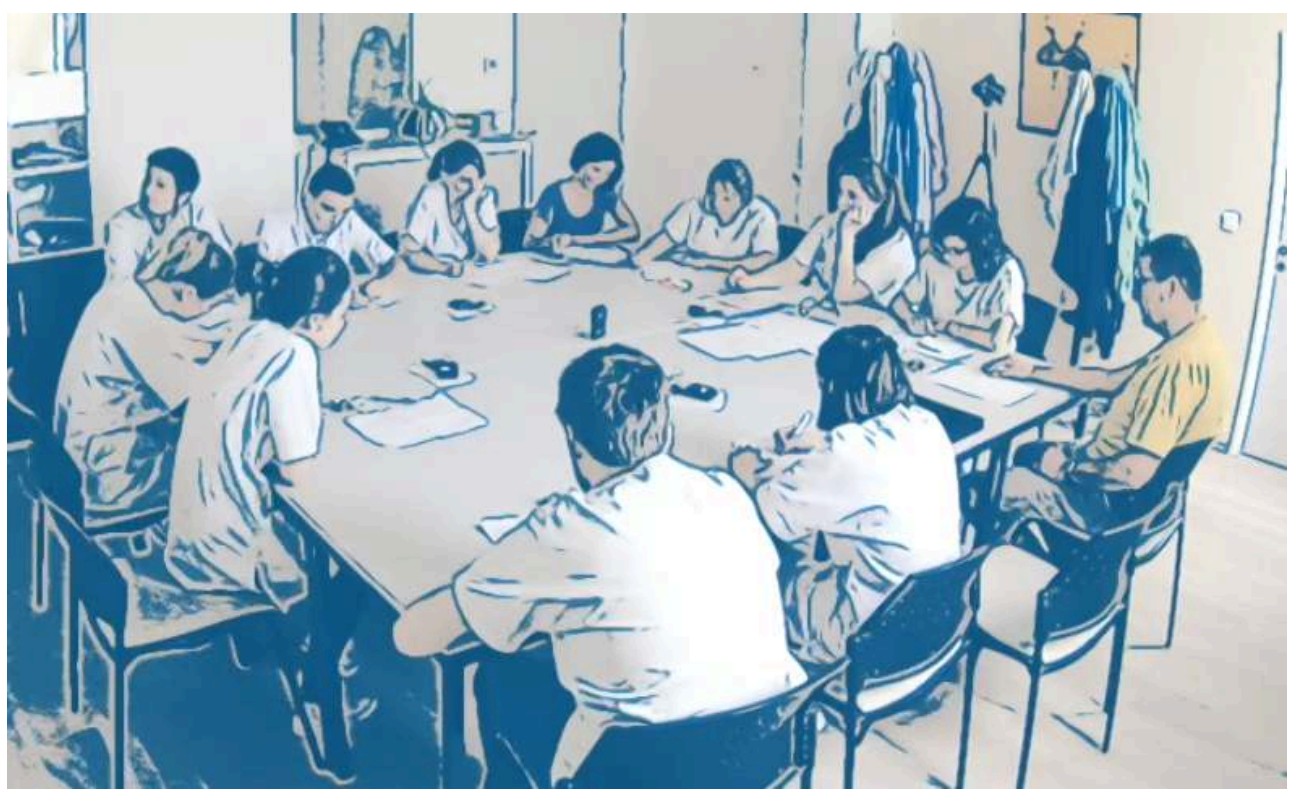

(c) Laboratoire ICAR (UMR5191)

Ligne 3, BER initie une séquence de réparation en mentionnant la patiente attendue. En réaction, PAC fait une pause et regarde GRI. Ligne 5, BER ré-initie la séquence de réparation, avec GRI en chevauchement qui en fait de même, en précisant l'origine de l'erreur, « à la douze » (versus la chambre 13 dont PAC énonce l'actualité clinique). PAC vérifie alors sur sa fiche de relève ( $c f$. image \#2-3) tandis que GRI la regarde, avant de produire une incise au cours de laquelle elle s'excuse (1. 7), puis redémarre la relève en auto-réparation, et décrit alors l'actualité clinique de madame Sarrazino, patiente de la chambre 12. On peut par ailleurs observer ici une nouvelle fois la structure syntaxique particulière "madame Sarrazino, qui... ». Lignes 8 à 18, alors que PAC décrit le weekend de madame Sarrazino, les autres participants notent les informations sur leurs feuilles de relève ( $c f$. image \#2-4). 


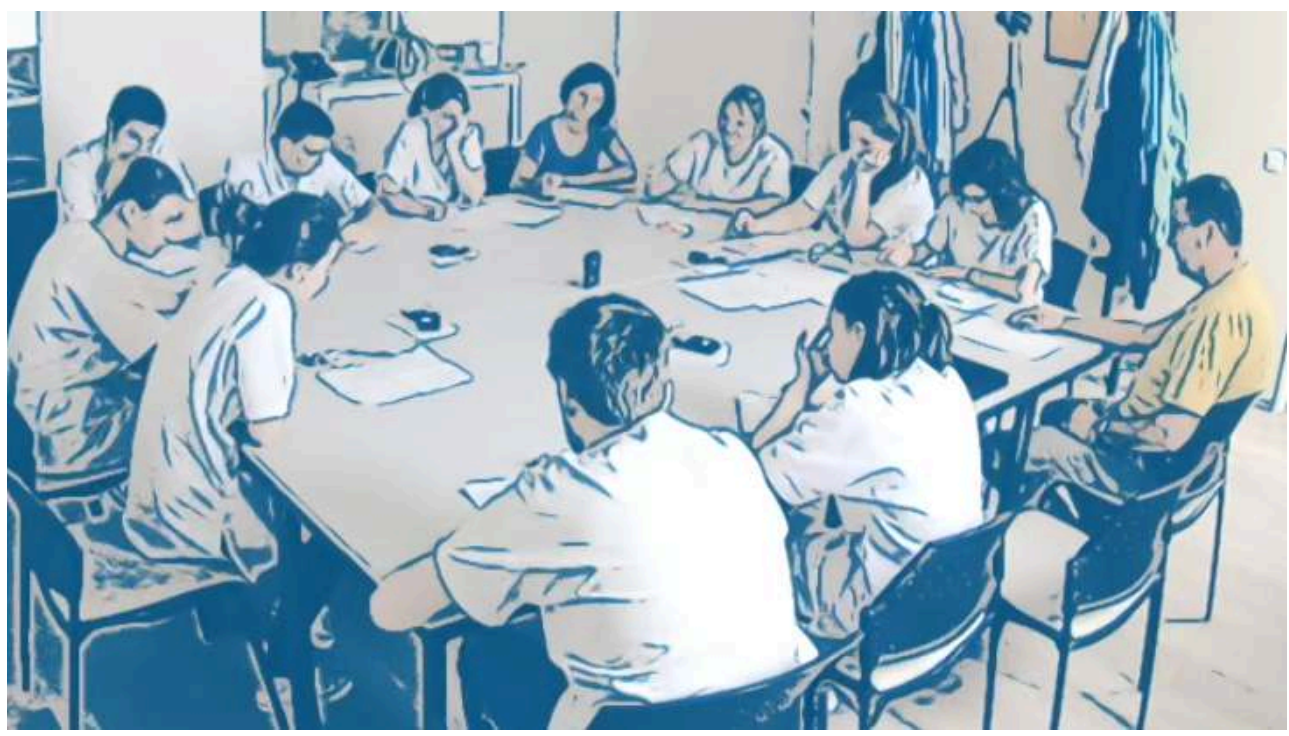

(c) Laboratoire ICAR (UMR5191)

\#2-4

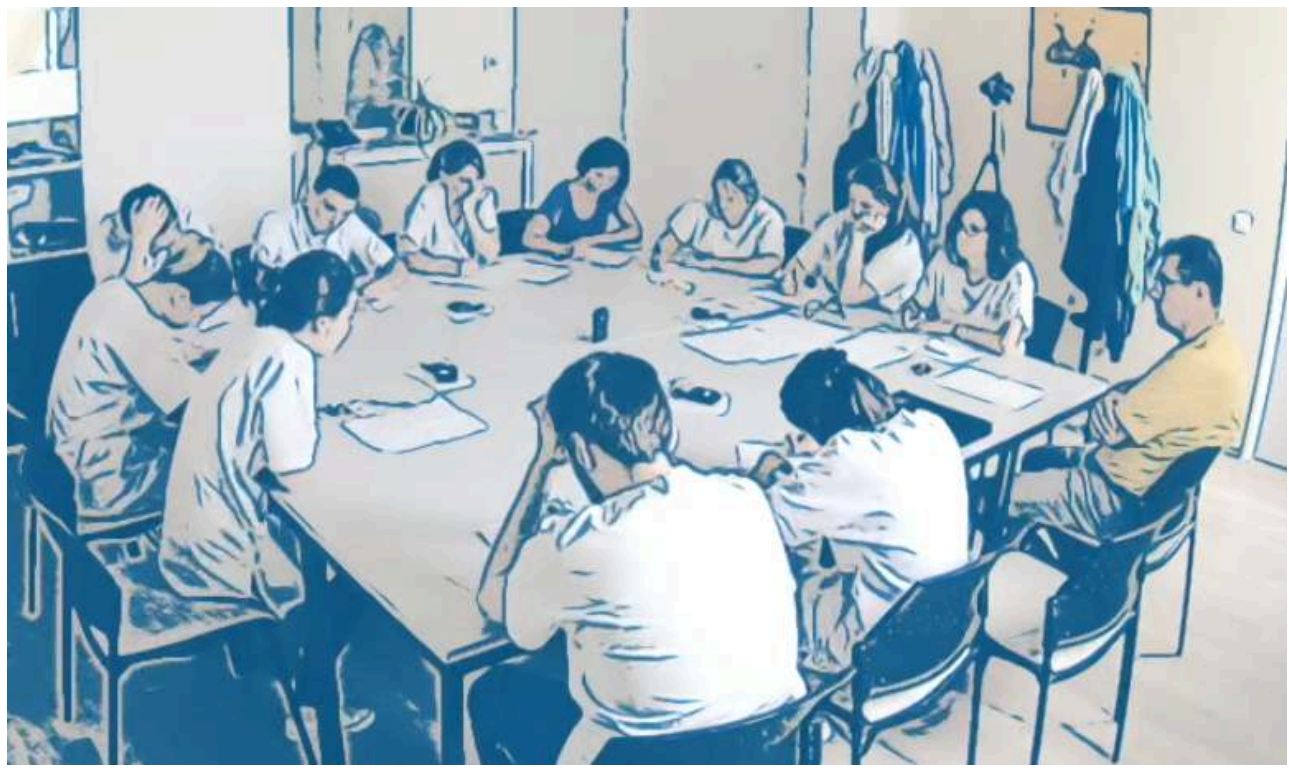

(c) Laboratoire ICAR (UMR5191)

41 Ligne 19, durant la pause marquée de 1,1 seconde, BER regarde PAC, puis voyant qu'elle a terminé son énoncé, prend la parole et saisit l'occasion du point de transition pour décrire son propre vécu face à la patiente. Cette alternance entre super locuteur et autres participants est également typique de notre corpus. Une fois les informations mutualisées de la part du super locuteur, les autres membres de la réunion peuvent prendre la parole et rendre audible à l'auditoire étendu leur propre expérience - leur propre savoir - vis-à-vis du patient dont il est question. Les regards se tournent alors vers $B E R$, et la prise de notes s'arrête pour les participants. Ce phénomène multimodal, au croisement des changements de propos (le super locuteur passe des informations globales aux informations personnellement vécues) et de postures (cessation d'activité 
de prise de notes et changement d'orientation du corps), est intéressant au regard de l'activité : les informations mutualisées par le super locuteur seront considérées comme primordiales, et par conséquent dignes d'être notées, alors que l'attention se portera préférentiellement sur l'écoute active de ce que les autres participants auront à dire de leur expérience personnelle. En effet, les réunions de relève sont des instants particuliers dans la vie du service, tant dans l'acquisition d'informations de la part des participants que dans le partage de ressentis de ces derniers. Là où l'actualité clinique pure sera décrite par le super locuteur, les autres infirmiers présents pourront partager des émotions vécues, une actualité plus focalisée sur un événement en particulier, ou même un ressenti, positif ou négatif face à une personnalité particulière.

On peut donc distinguer deux rôles particuliers du point de vue informationnel : le super locuteur, comme porteur du "faire savoir », décrivant une actualité nécessaire au bon déroulement des soins au sein de la structure, et les autres participants, comme prenant en charge le "faire entendre ", qu'il s'agisse des paroles du patient ou de ses propres paroles ou pensées. Cependant, il convient de noter que les postures au sein des réunions de relèves ne se cantonnent pas à ces deux dernières. Les deux extraits suivants en montrent une autre, le «faire apprendre ». En effet, comme nous allons le voir, ces réunions auxquelles est présente une grande partie de l'équipe du service sont aussi un lieu d'apprentissage actif, et de formation continue dans la pratique de soins infirmiers.

\section{6. « Faire apprendre » pendant le partage de connaissance professionnelle}

43 L'extrait 3 réunit six participants dont seulement quatre prendront la parole pendant cette séquence, 3 infirmières, COU, GIL et PAT, et un aide-soignant, TAG. La relève a lieu à $6 \mathrm{~h}$ entre l'équipe de nuit (COU et TAG) et celle du matin (GIL et PAT), elle est conduite par COU. L'extrait commence lorsqu'ils ont terminé l'actualité des patients chambre par chambre et s'orientent vers la clôture de la relève. COU en profite pour introduire une question sur " les eaux gélifiées » et savoir s'il y en a d'autres en stock (l. 1). PAT n'attend pas la fin du tour de COU pour répondre et préciser qu'elle en a commandé (l. 2). En chevauchement de son tour également, COU et TAG notifient tous les deux la réception de cette information (1. 4-5) et TAG va ajouter une nouvelle information " $j$ " les ai mis au frigo » (1.5-6) qui va susciter un moment d'apprentissage partagé entre les participants leur permettant d'améliorer leur pratique professionnelle. 


\section{Extrait 3 : eau gélifiée}

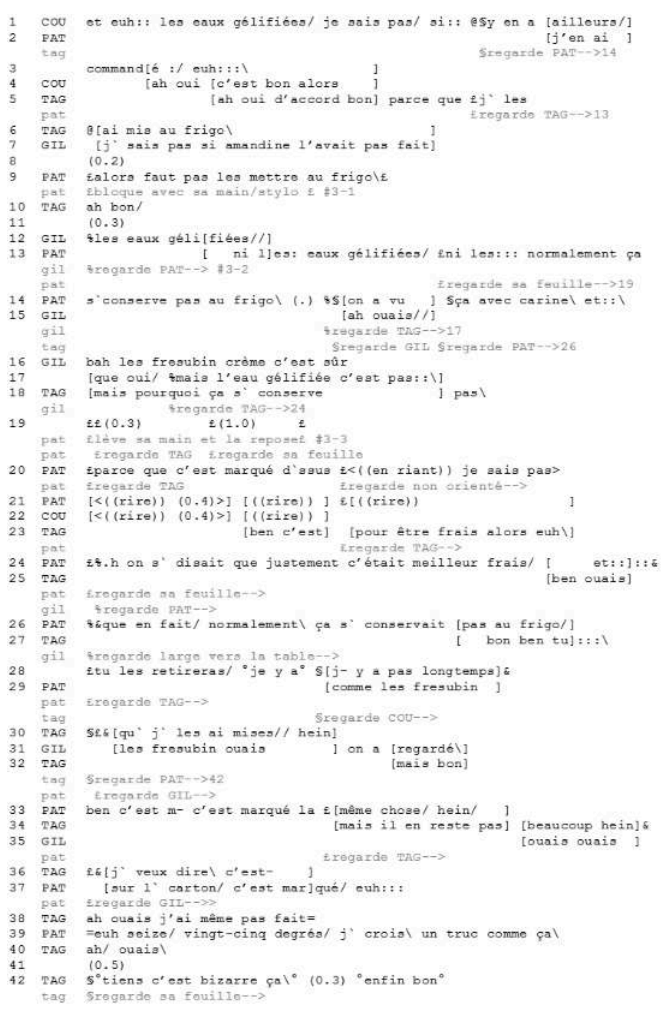

A la ligne 9, PAT va réagir et partager un contenu concernant la pratique professionnelle des soignants : «alors faut pas les mettre au frigo\». Son discours est accompagné d'un geste bref et marqué de sa main tenant un stylo (cf. image \#3-1) appuyant ainsi la recommandation donnée à l'oral. En effet, après avoir levé son stylo, elle le baisse très rapidement vers la table, ce geste étant synchrone avec "pas", appuyant ainsi l'obligation. Les orientations des regards entre PAT et TAG sont alors réciproques, ils se regardent mutuellement. Bien que l'information de PAT s'adresse particulièrement à TAG étant donné qu'il s'agit du soignant ayant mal stocké les eaux gélifiées (lignes 5-6), celle-ci est entendue par tous ce qui va faire émerger plusieurs interrogations de la part d'autres participants. On voit en effet qu'aux lignes 10 et 12, TAG et GIL réagissent avec étonnement à cette nouvelle information " ah bon/ ", « les eaux gélifiées// ». Le bon mode d'emploi des « eaux gélifiées » ne semble donc pas être connu de tous, que ce soit dans l'équipe de nuit ou dans celle de jour, puisqu'un soignant de chacune des deux équipes partage son étonnement.

\#3-1
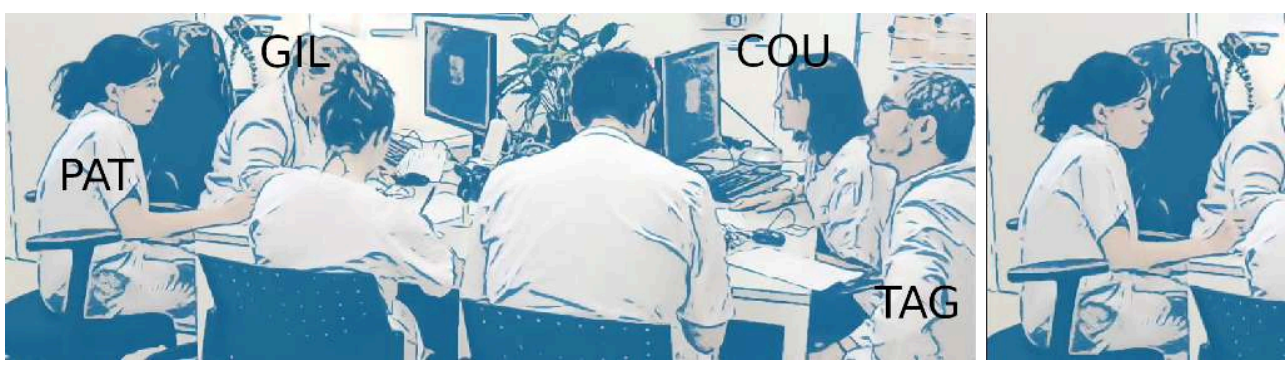

(c) Laboratoire ICAR (UMR5191)

TIPA. Travaux interdisciplinaires sur la parole et le langage, 36 | 2020 
PAT explique qu'elle a vérifié cette information « avec Carine » (1. 14). Même si tous les participants ne prennent pas part oralement à l'échange en cours, on observe, entre le début et la fin du tour de PAT (1. 13-14), une orientation brève mais collective des regards ${ }^{4}$ des cinq soignants dans sa direction (cf. image \#3-2). Multimodalement, il est aussi intéressant de remarquer que dans cet extrait, la prise de notes sur les feuilles de relève ne semble plus pertinente pour les participants. Ils sont à présent engagés dans un partage de connaissance professionnelle qui n'est pas propre à un patient en particulier.

\#3-2

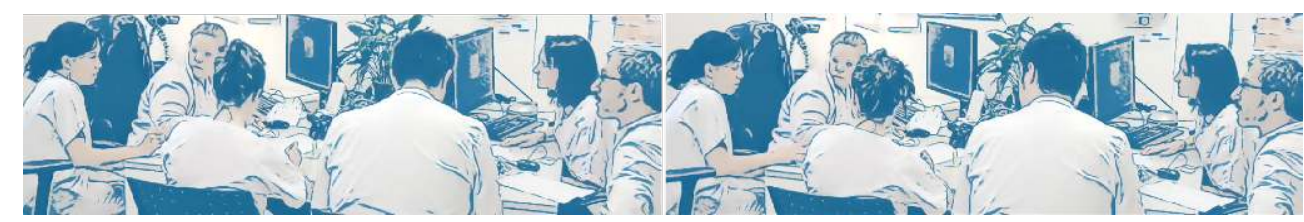

(c) Laboratoire ICAR (UMR5191)

TAG et GIL, qui avaient déjà réagi avec étonnement aux tours précédents, continuent de s'interroger sur cette nouvelle pratique à adopter dans leur travail: GIL met en comparaison l'eau gélifiée avec un autre produit, le fresubin, dont elle est sûre qu'il ne se conserve pas au frigo alors que TAG, en chevauchement de son tour, demande " pourquoi ça s` conserve pas\» (l. 16-18). Pendant une longue pause de 1.2 secondes (l. 19), PAT oriente son regard successivement entre TAG et sa feuille, tout en effectuant un geste de sa main ouverte de bas en haut pour la reposer sur la table (cf. image \#3-3). Son geste anticipe sa réponse à la ligne 20 lorsqu'elle apporte une nouvelle raison d'appliquer cette pratique professionnelle: "parce que c'est marqué d'ssus <((en riant)) je sais pas » ". Rétrospectivement, on peut analyser le geste à la ligne 19 comme un geste communicatif quasi linguistique d'après Cosnier \& Vaysse (1997), geste qui indique de façon conventionnelle l'incertitude de la nouvelle explication donnée à la ligne 20. En effet, le produit en question n'est pas à proprement parler un médicament, il s'agit simplement d'eau à laquelle on ajoute des gélifiants, pour éviter aux patients présentant des problèmes de déglutition un risque d'étouffement en buvant. Ainsi, médicalement parlant, il n'y a pas de raison apparente de devoir conserver lesdits produits à température ambiante, et les co-participants TAG et GIL ne se contentent pas d'apprendre qu'il ne faut pas mettre l'eau gélifiée au frigo.Ils veulent en connaître la raison, l'explication précise, ce qui met PAT en position de devoir justifier son information plus en détail, ce qu'elle n'est pas en mesure de faire. 


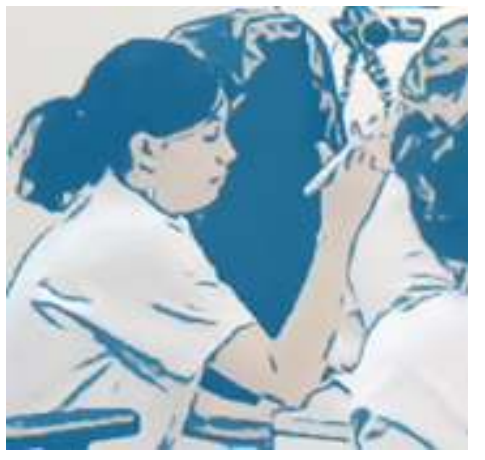

(c) Laboratoire ICAR (UMR5191)

PAT poursuit son tour par un rire partagé avec COU (1. 21-22). Du point de vue de PAT, son rire vient atténuer son incertitude évoquée en disant «je sais pas » et préserver ainsi sa face (Goffman, 1973 ; Brown \& Levinson, 1978/1987), mais ne remet pas en question pour autant son information initiale donnée (i.e. l'eau gélifiée ne se met pas au frigo). En chevauchement de son rire, TAG cherche toujours à comprendre et donne l'argument de la fraîcheur de l'eau gélifiée si elle est au frigo (1. 23). PAT s'aligne sur cet argument donné (1.24) mais malgré cela, l'eau gélifiée ne doit pas être conservée au frigo (1. 26). L'extrait se termine par une forme d'acceptation de la part de TAG qui ne cherche plus à savoir la raison précise auprès de PAT. Il demande à ses collègues de retirer les poches d'eau gélifiée déposées dans le frigo (1. 27-28). Quant à GIL, elle compare de nouveau avec le fresubin, un autre produit (mentionné par PAT, ligne 29) que les soignants doivent conserver hors du frigo. Un bref échange a lieu entre GIL et PAT sur le sujet (observable par l'orientation des regards mutuels entre elles) et cette dernière apporte enfin une information plus précise qui vient compléter la raison donnée à la ligne 20 ("parce que c'est marqué ») : elle explique que "sur l' carton/ c'est marqué ((---)) seize/ vingt-cinq degrés/ j’ crois un truc comme ça \» $(1.37,39)$. Après cette séquence de "faire apprendre ", l'ensemble des participants s'orientent enfin vers COU qui clôture la relève du matin.

L'analyse de l'extrait 3 nous a permis de montrer l'organisation multimodale de l'interaction pendant une séquence émergente de "faire apprendre » entre soignants qui se traduit dans ce cas par de nombreuses (ré-)orientations des regards entre les participants et deux gestes qui viennent appuyer le discours à portée didactique du soignant en charge d'une nouvelle connaissance professionnelle. Un dernier extrait va nous permettre de montrer plus en détail la richesse des ressources multimodales mobilisées par un soignant lors d'une séquence d'explication théorique sur l'homéopathie pendant une relève.

L'extrait 4 réunit huit participants, dont cinq prendront part à l'échange analysé : GIL (l'infirmière en charge de la relève), BER, PAT, ALI et PAC. Seule ALI est aide-soignante, les quatre autres sont infirmières. Nous sommes à la relève de $14 \mathrm{~h}$ et l'actualité des patients est en cours de développement. GIL est en train de partager des informations sur une patiente qui prend un placebo tous les deux jours le soir pour dormir. Elle informe ses collègues qu'un nouveau traitement à base d'euphytose va être prescrit sur ordonnance. BER s'interroge alors sur la pertinence de ce traitement qui pour elle est un traitement « actif " par rapport à un placebo. Elle explique que l'euphytose n'est pas de l'homéopathie, et par conséquent, ce n'est pas un placebo. PAT réagit en précisant 
que l'homéopathie «agit» aussi (sous-entendu comme l'euphytose). BER insiste en disant que "l'euphythose c'est des plantes » alors que "l'homéopathie c'est rien c'est que du sucre », permettant malgré tout l'effet placebo qui reste efficace. Un désaccord s'installe entre PAT et BER : l'une dit qu'il y a des plantes dans l'homéopathie alors que l'autre affirme le contraire. Ce désaccord va ouvrir une longue séquence de «faire apprendre » sur ce qu'est l'homéopathie, expliquée par BER, et pourquoi l'homéopathie peut être considérée comme placebo.

\section{Extrait 4 : homéopathie}

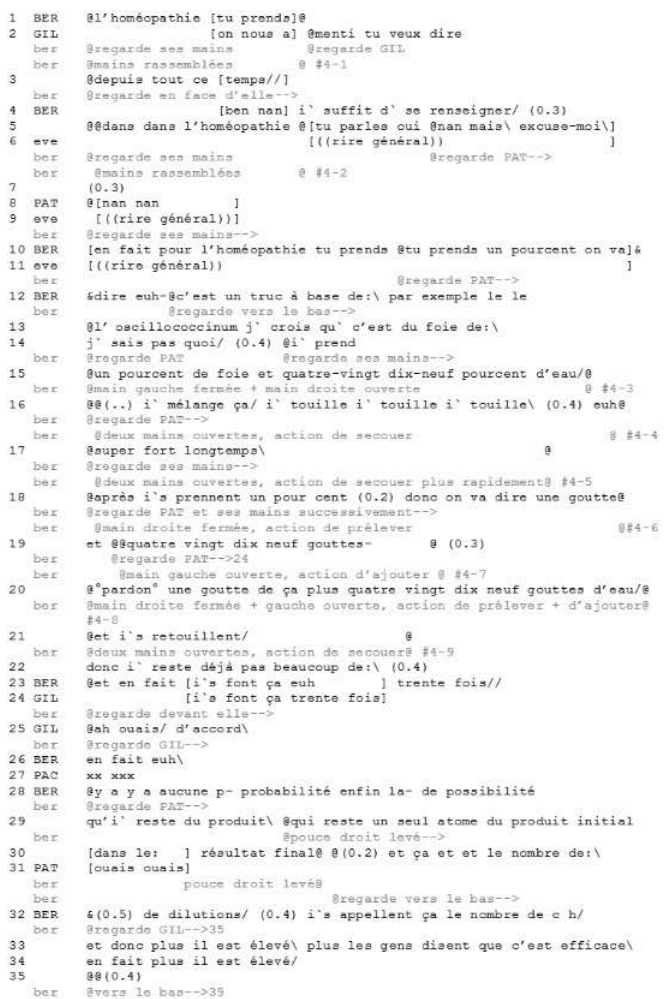

Ligne 1, BER initie son explication théorique de ce qu'est l'homéopathie en rassemblant ses mains en face d'elle en l'air ( $c f$. image \#4-1) mais elle est tout de suite interrompue par GIL qui s'exclame de façon humoristique sur le fait qu'il n'y aurait pas de plantes dans l'homéopathie (1. 2-3). BER répond directement «ben nan i' suffit de se renseigner/ » (1. 4) et enchaîne sur la reprise de son explication en répétant le geste de ses mains rassemblées en face d'elle ( $c f$. image \#4-2). Mais son tour précédent suscite un rire général de la part de tous les co-participants (l. 6) ce qui amène BER à s'interrompre de nouveau pour s'excuser rapidement auprès de PAT vers qui elle oriente son regard en même temps (1. 5). Pendant que les rires se poursuivent, BER réoriente son regard vers ses mains et montre ainsi son ré-engagement dans l'activité de produire son explication sur l'homéopathie (1. 8-9), ce qu'elle fait à la ligne 10, toujours en chevauchement des rires collectifs. En fin de tour, elle oriente de nouveau son regard vers PAT. Les rires s'arrêtent et les participants maintiennent leurs regards collectifs orientés vers BER. 


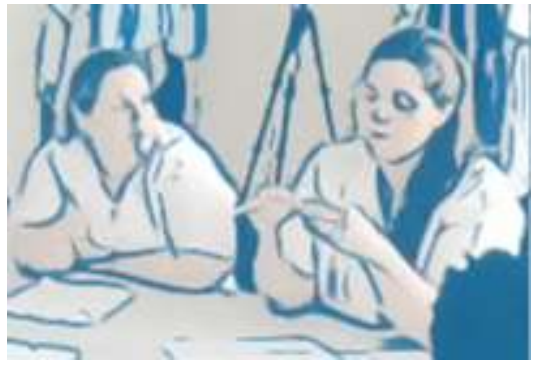

(c) Laboratoire ICAR (UMR5191)

\#4-2

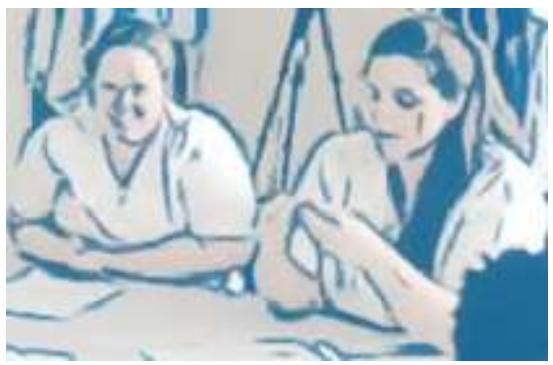

(c) Laboratoire ICAR (UMR5191)

BER prend exemple sur un médicament homéopathique connu de tous, l'oscillococcinum, qui contient $\mathrm{du}$ foie de canard (1. 12-14). Elle explique que les fabricants prennent « un pour cent de foie et quatre-vingt-dix-neuf pour cent d'eau/ ». A ce discours, elle y associe des gestes illustrant son propos et temporellement alignés aux termes utilisés : la main droite est ouverte (symbolisant les $99 \%$ d'eau) et la main gauche est fermée (illustrant les $1 \%$ de foie) (cf. image \#4-3). À ce moment-là, elle regarde alors ses mains. Elle explique qu'il faut ensuite mélanger et touiller (répété trois fois, 1. 16). Le terme "touiller" employer au registre familier, semble ici venir discréditer l'approche scientifique de l'homéopathie. Multimodalement, elle réoriente son regard vers PAT et secoue ses deux mains ouvertes (cf. image \#4-4). Elle ajoute encore une information qui vient caractériser l'action de mélanger: «super fort longtemps» (1. 17) en regardant de nouveau ses deux mains (cf. image \#4-5) qu'elle secoue plus rapidement que précédemment.

\#4-3

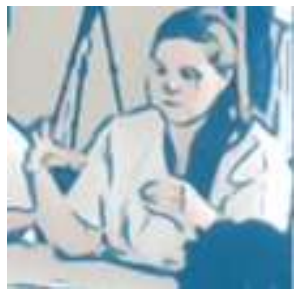

(C) Laboratoire ICAR (UMR5191) 


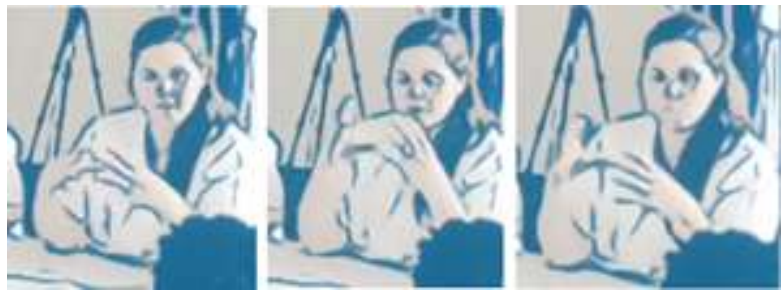

(c) Laboratoire ICAR (UMR5191)

\#4-5

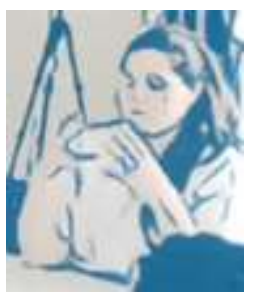

(c) Laboratoire ICAR (UMR5191)

Elle passe à l'étape suivante du processus qui est de prendre « un pour cent $(0.2)$ donc on va dire une goutte» (1. 18). Elle regarde PAT puis rapidement regarde de nouveau ses mains. Gestuellement, sa main droite est fermée et représente l'action de prélever quelque chose (mouvement de bas en haut) (cf. image \#4-6). Elle répète ce geste deux fois de suite, une première fois sur « un pour cent » et une seconde fois sur « on va dire une goutte». Puis elle complète son tour en ajoutant "et quatre-vingt-dix-neuf gouttes- $(0.3)$ » tout en regardant PAT. Sa main gauche est alors ouverte et représente l'action d'ajouter quelque chose (vers la main droite) (cf. image \#4-7). Cependant, elle s'interrompt dans son tour et dans ses gestes pour répéter l'ensemble au tour suivant.

\#4-6
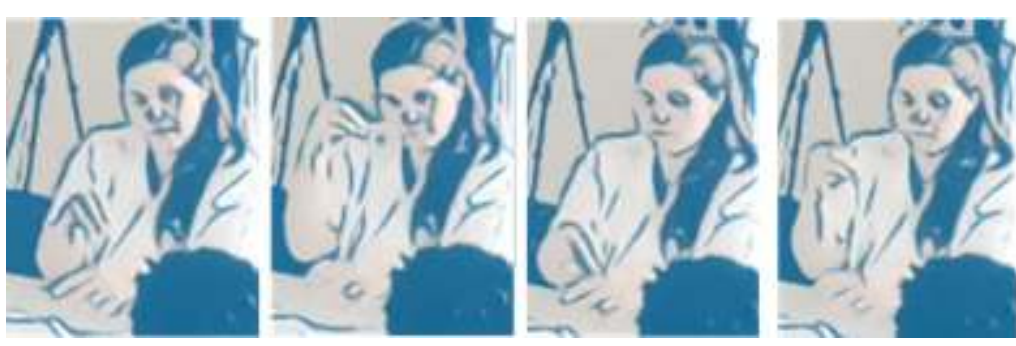

(c) Laboratoire ICAR (UMR5191) 


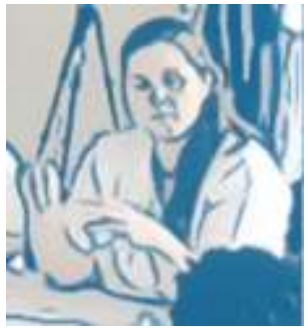

(c) Laboratoire ICAR (UMR5191)

En effet, à la ligne 20, elle répète le processus « une goutte de ça plus quatre-vingt-dixneuf gouttes d'eau/». Elle ajoute ici le terme de l'eau. L'enchaînement des gestes est identique au tour précédent : la main droite est fermée alors que la gauche est ouverte. La main droite représente l'action de prélever alors que la main gauche représente l'action d'ajouter (cf. image \#4-8). Elle explique que de nouveau «i`s retouillent » (1. 21) et encore une fois le geste est identique au tour similaire (1.16-17) : les deux mains sont ouvertes et elle les secoue de soi vers le bas (cf. image \#4-9). En touillant verticalement, elle renvoie à une action industrielle intense en opposition à une action horizontale de "mélange" qui est plus courante et "délicate» en cuisine. Par ce processus, elle commence à montrer qu'il ne reste pas beaucoup de plantes au final (1. 22). Elle précise que les fabricants répètent ce processus « trente fois » (1.23) et que par conséquent, il y a " aucune possibilité qui reste un seul atome du produit initial dans le résultat final » (1. 28-30).

\#4-8

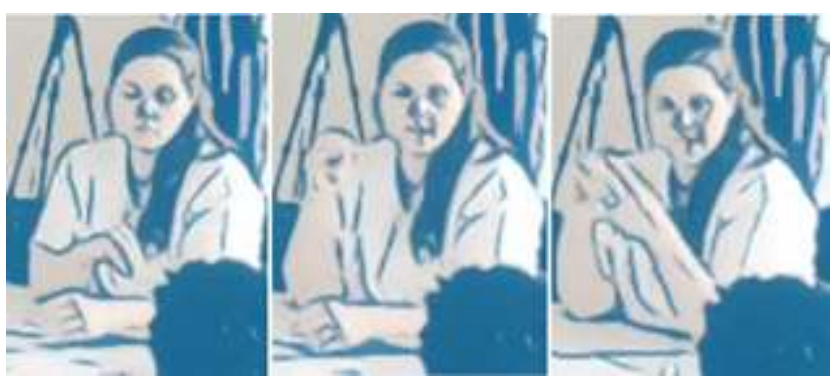

(c) Laboratoire ICAR (UMR5191)

\#4-9

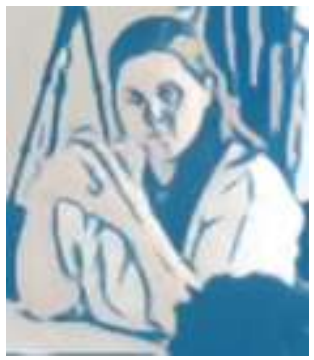

(c) Laboratoire ICAR (UMR5191)

Elle conclut son explication en indiquant que le résultat final devient donc « le nombre de dilutions» $(1.30,32)$ et qu'il s'agit du «nombre de $c h »(1.32)$ et partage une idée 
reçue selon laquelle «plus il est élevé \plus les gens disent que c'est efficace \» (l. 33). Elle termine son énoncé par un tour inachevé, ligne 34, marqué par une intonation montante "en fait plus il est élevé/ » et suivi d'une pause, qui laisse place aux coparticipants pour compléter son tour. GIL et ALI s'auto-sélectionnent simultanément pour terminer le tour de BER (1.36-37). La place transitionnelle laissée pendant la pause est aussi multimodalement marquée par un geste de BER qui, les mains et bras ouverts, effectue un mouvement de bas en haut pour revenir en bas, tout en souriant et en regardant vers le bas ( $c$ f. image \#4-10). Son geste vient renforcer son explication dans le sens où si on a compris le processus de dilution réalisé dans l'homéopathie qu'elle vient de détailler, on comprend que le pourcentage de plantes est infime et qu'il s'agit bien d'un placebo.

\#4-10

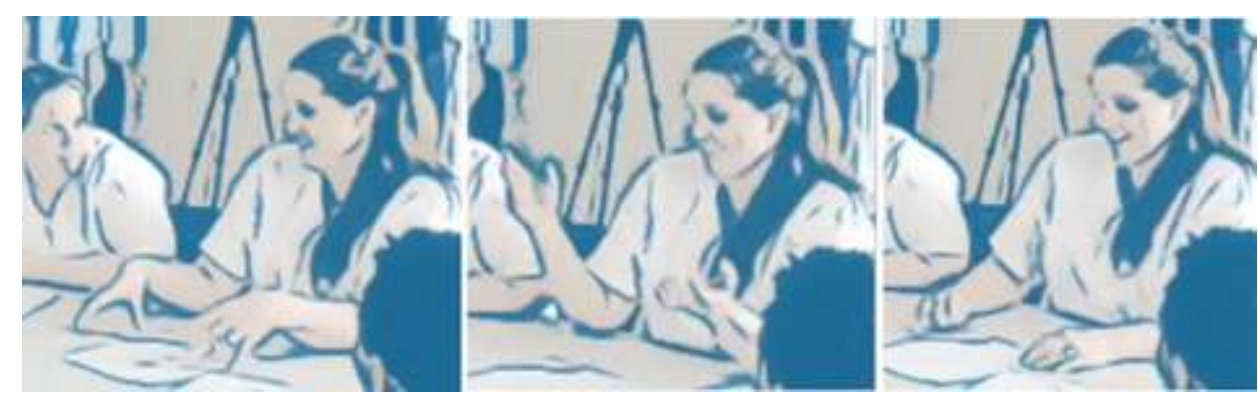

(C) Laboratoire ICAR (UMR5191)

La séquence concernant le placebo de la patiente se termine sur la façon éthique et légale de prescrire le traitement. On peut souligner un dernier tour non représenté dans la transcription mais qui conclut cette longue séquence sur l'homéopathie. Il s'agit du tour de GIL qui remercie BER pour « le cours ». Du point de vue des participants, le moment a été par conséquent perçu comme didactique. Comme dans l'extrait 3, l'organisation multimodale de cette interaction didactique s'oriente plus vers de nombreuses alternances de regards entre les co-participants (qui eux restent le regard tourné vers $\mathrm{BER}$ ), ses mains et la table, ainsi que vers des gestes ici répétés et précis. La prise de notes sur les feuilles de relève n'est encore une fois plus pertinente pour les soignants. On sort de l'actualité précise de la patiente pour partager des connaissances plus générales (ici sur l'homéopathie) qui peuvent servir les soignants dans leur pratique professionnelle.

\section{Conclusion}

La relève infirmière, liant des équipes de soins en psychiatrie, garante de la continuité des soins, est riche d'une interaction singulière et variée et ne se limite pas à la simple transmission d'informations, mais tend à co-construire une réflexion d'équipe tournée vers le patient.

57 L'approche interactionnelle et multimodale proposée ici vient mettre en lumière des compétences spécifiques de l'exercice infirmier au quotidien. Dans l'annexe 1 de l'arrêté du 31 juillet 2009 relatif au diplôme d'État d'infirmier du Ministère de la Santé et des sports, les transmissions d'informations sont exclusivement décrites comme prenant leur source dans l'actualité clinique du patient, pour ensuite être dirigées vers 
les équipes soignantes ainsi que les patients et leurs familles. Nous avons cependant pu voir que lors des réunions de relève, lieux d'échanges inter-équipes par excellence dans l'exercice du soin infirmier en psychiatrie, l'actualité clinique du patient, autrement dit le « faire savoir ", n'est pas la seule préoccupation des soignants.

Si l'on peut observer une forte tendance à ne décrire que l'état psychologique et somatique du patient, une dimension didactique apparaît de façon assez fréquente, en rapport avec l'exercice infirmier en tant que tel, et non plus directement avec les patients.

Du point de vue multimodal, ces moments que nous qualifions de "faire apprendre " sont repérables par des postures spécifiques. Durant les séquences de "faire savoir ", les soignants auront tendance à noter sur leur feuille de relève les informations données par le super locuteur en charge de l'énonciation de l'actualité clinique des patients, tandis que durant les phases didactiques de "faire apprendre ", les regards auront tendance à s'orienter vers le locuteur transmettant les savoirs et compétences, et l'activité d'écriture cessera. En ce qui concerne le locuteur prenant en charge l'énonciation du " faire apprendre ", nous avons pu noter une forte gestualisation des propos tenus, comme le montre également Filliettaz $(2005,2009)$ dans ses travaux sur les réunions de relève de poste en milieu industriel, qui supporte le discours ou marque un décrochement séquentiel différenciant les phases de « faire apprendre » et de « faire savoir ».

60 Enfin, la coordination multimodale typique des relèves infirmières désigne ou appuie une position de super locuteur de l'infirmier en charge de la relève. Cette compétence spécifique décrite ici fait état de la rigueur d'énonciation des patients présents dans le service, de la capacité de synthétiser ses propos, tout en restant attentif au groupe soignant, celui-ci ayant un rôle contrôle du bon déroulement des séquences, tout en venant enrichir les propos énoncés, ou en demandant des éclaircissements.

61 Le projet CIPSY permet ainsi de mettre en avant une richesse " technique " de l'infirmier à travers des compétences spécifiques décrites ici. Les analyses futures du corpus complexe constitué viendront illustrer la richesse du travail quotidien des infirmiers, l'analyse conversationnelle y trouvant ici un terrain d'exploration nouveau et contributif à la qualité des soins. La collaboration du soin et des sciences du langage, dans la lignée des travaux de Grosjean \& Lacoste (1999), tend donc à préciser en quoi les réunions de transmissions inter-équipes amènent à « compléter des savoirs » ou « aider le partenaire à y voir clair » (1999 : 12), qu'il s'agisse de l'actualité clinique des patients (relevant donc d'un " faire savoir ", cf. partie 1) ou des compétences requises dans l'exercice infirmier (relevant d'un « faire apprendre », cf. partie 2).

Les auteurs remercient le LABEX ASLAN (ANR-10-LABX-0081) de l'Université de Lyon pour son soutien financier dans le cadre du programme "Investissements d'Avenir " (ANR-11-IDEX-0007) de l'État Français géré par l'Agence Nationale de la Recherche (ANR). Ils remercient également le Centre hospitalier le Vinatier, qui dans le cadre d'un partenariat entre le Comité Scientifique de la Recherche et l'Université Lyon 2, participe au financement de cette étude. 


\section{BIBLIOGRAPHIE}

Abraham, J., Kannampallil, T., Brenner, C., Lopez, K.D., Almoosa, K.F., Patel, B. \& V.L. Parel (2015) Characterizing the structure and content of nurse handoffs: A Sequential Conversational Analysis approach, Journal of Biomedical Informatics, 59, p.76-88.

Bangerter, A., Mayor, E. \& S. Pekarek Doehler (2011) Reported speech in conversational storytelling during nursing shift handover meetings, Discourse Processes, 48, 3, p. 183-214.

Brown, P. \& S.C. Levinson (1978/1987) Politeness: Some Universals in Language Usage, Studies in Interactional Sociolinguistics, 4, Cambridge: Cambridge University Press.

Cosnier, J. \& J. Vaysse (1997). Sémiotique des gestes communicatifs, Nouveaux actes sémiotiques, vol. 52, p. 7-28.

Duncan, S. \& D. W. Fiske (2015 [1977]) Face-to-face interaction. Research, Methods and Theory, London: Routledge.

Efron, D. (1941) Gesture and environment, Oxford, England: King's Crown Press.

Ekman, P. \& Friesen, W.V. (1967) The repertoire of non-verbal behavior, Semiotica, 1, p. 49-98.

Filliettaz, L. (2005) Gestualité et (re)contextualisation de l'interaction dans des réunions de relève de poste en milieu industriel [en ligne], Actes du colloque Interacting bodies / Le corps en interaction, Lyon : Université Lumière Lyon 2. Disponible sur : https://www.unige.ch/fapse/interactionformation/files/6214/2608/6877/Filliettaz-Gestualite-et-recontextualisation.pdf.

Filliettaz, L. (2009) Les représentations du travail dans des réunions de relève de poste en milieu industriel. Une analyse multimodale, in Canelas-Trevisi, S., M.-C. Guernier, G. Sales Cordeiro \& D. Lee Simon (eds) Langage, objets enseignés et travail enseignant, Grenoble: ELLUG, p. 47-70.

Garfinkel, H. (1967) Studies in ethnomethodology, Cambridge: Polity Press.

Goffman, E. (1973) La mise en scène de la vie quotidienne. Les relations en public, tome 2, Paris: Minuit. Goodwin, C. (1980) Restarts, pauses, and the achievement of a state of mutual gaze at turnbeginning, Sociological Inquiry, 50, 3-4, p. 272-302.

Goodwin, C. (2000) Gesture, aphasia, and interaction, Language and gesture, 2, p. 84-98.

Grosjean, M. \& M. Lacoste (1999) Communication et intelligence collective, Paris: Presses Universitaires de France.

Grosjean, M. (1997) Les relèves orales entre équipes infirmières à l'hôpital. Des structurations discursives aux pratiques cognitives et sociales, in Grossen, M. \& B. Py (eds) Pratiques sociales et médiations symboliques, Berne: Peter Lang, p.137-152.

Grosjean, M. (2004) From multi-participant talk to genuine polylogue: shift-change briefing sessions at the hospital, Journal of Pragmatics, 36, 1, p.25-52.

Groupe ICOR (2006) La démarche ethnographique, Corinte [en ligne]. Disponible sur : http:// icar.cnrs.fr/projets/corinte/recueil/demarche_ethnographique.htm (consulté le 15 juillet 2019). Haddington, P. (2006) The organization of gaze and assessments as resources for stance taking, Text \& Talk, 26, 3, p. 281-328. 
Heath, C. (2018) Embodying Action: Gaze, Mutual Gaze and the Revelation of Signs and Symptoms during the Medical Consultation", in Favareau, D. (ed.), Co-operative Engagements in Intertwined Semiosis: Essays in Honour of Charles Goodwin, Tartu: University of Tartu Press, p. 164-177.

Kendon, A. (2004) Gesture: Visible Action as Utterance, New York: Cambridge University Press.

Lanza, D., Longchamp, P., Seferdjeli, L. \& R. Müller (2004) Entre paroles et actions, appréhender la complexité de la coopération en milieu hospitalier, Langues de l'hôpital, pratiques communicatives et pratiques de soins, Cahiers de l'ILSL, 16, p.97-129.

LeBaron, C., Christianson, M. K., Garrett, L. \& R. Ilan (2016) Coordinating Flexible Performance During Everyday Work: An Ethnomethodological Study of Handoff Routines, Organization Science, 27, 3, p. 514-534.

MacWhinney, B. (2000) The CHILDES Project: Tools for Analyzing Talk. 3rd ed., Mahwah, NJ: Lawrence Erlbaum Associates.

McNeill, D. (1992) Hand and mind: What gestures reveal about thought, Chicago: University of Chicago Press.

Merkling, J. (2007) Le métier d'infirmier en santé mentale : savoirs et pratiques, Paris : Seli Arslan.

Moirand, S. (1993) Autour de la notion de didacticité [en ligne], Les Carnets du Cediscor. Disponible sur : http://journals.openedition.org/cediscor/600 (mis en ligne le 28 août 2009, consulté le 02 juillet 2020).

Mondada, L. (2014) The local constitution of multimodal resources for social interaction, Journal of Pragmatics, 65, p. 137-156.

Mondada, L. (2018) Multiple Temporalities of Language and Body in Interaction: Challenges for Transcribing Multimodality, Research on Language and Social Interaction, 51, 1, p. 85-106.

Mori, J., Imamura, A. \& C. Shima (2017) Epistemic management in the material world of workplace: A study of nursing shift handovers at a Japanese Geriatric Healthcare Facility, Journal of Pragmatics, 109, p. 64-81.

Psathas, G. (1990) The organization of talk, gaze, and activity in a medical interview, in Psathas, G. (ed.) Interaction Competence, Washington: University Press of America, p. 205-230.

Rossano, F. (2013) "Gaze in conversation", in Sidnell, J., \& T. Stivers (eds),The Handbook of Conversation Analysis, Oxford, U.K.: Wiley-Blackwell, p. 308-329.

Sacks, H. (1992) Lectures on conversation, vol. 1 et 2, Oxford: Basil Blackwell.

Sacks, H., Schegloff, E. A. \& G. Jefferson (1974) A simplest systematics for the organization of turn-taking for conversation, Language, 50, p. 696-735.

Schegloff, E.A. \& H. Sacks (1973) Opening up closings, Semiotica, 8, p. 289-327.

Sidnell, J. (2006) Coordinating Gesture, Talk, and Gaze in Reenactments, Research on Language and Social Interaction, 39, 4, p. 377-409.

Streeck, J. (2014) Mutual gaze and recognition: Revisiting Kendon's 'Gaze direction in two-person conversation', in Seyfeddinipur, M. \& M. Gullberg (eds.), From Gesture in Conversation to Visible Action as Utterance: Essays in Honor of Adam Kendon, Amsterdam / Philadelphia: John Benjamins, p. 35-56. 
Wittenburg, P., Brugman, H., Russel, A., Klassmann, A. \& H. Sloetjes (2006) ELAN: a Professional Framework for Multimodality Research, in Proceedings of LREC 2006, Fifth International Conference on Language Resources and Evaluation.

\section{ANNEXES}

\section{Conventions de transcription}

\begin{tabular}{|l|l|}
\hline[] & Chevauchement de paroles \\
\hline$/ \backslash$ & Intonations montantes ou descendantes \\
\hline 00 & Voix murmurée \\
\hline$:::$ & Allongement d'un son ou d'une syllabe \\
\hline p`tit & Elision \\
\hline trouv- & Troncation d'un mot \\
\hline xxx & Syllabe incompréhensible \\
\hline$=$ & Enchaînement immédiat \\
\hline() & Transcription incertaine \\
\hline$(())$ & Commentaires \\
\hline$\&$ & Tour du même locuteur interrompu par un chevauchement \\
\hline$()$. & Micro-pause \\
\hline$(0.6)$ & Pause quantifiée \\
\hline
\end{tabular}

Plus de détails : http://icar.univ-lyon2.fr/projets/corinte/documents/ 2013_Conv_ICOR_250313.pdf

La transcription de la parole est en police noire

La transcription multimodale est en police grise

Les conventions de transcription de la multimodalité sont reprises de Mondada (2018) :

Les descriptions multimodales sont temporellement alignées aux tours de parole à l'aide de symbole.

\begin{tabular}{|l|l|}
$@$ ou $£$ ou $\S$ & $\begin{array}{l}\text { Un symbole est associé à chaque locuteur pour décrire les regards et les gestes } \\
\text { réalisés lorsqu'ils sont pertinents pour l'analyse. }\end{array}$
\end{tabular} 


\begin{tabular}{|l|l|}
\hline $\begin{array}{l}\text { @regarde } \\
\text { GIL@ }\end{array}$ & On note le début et la fin du phénomène multimodal par le même symbole. \\
\hline$-->$ & $\begin{array}{l}\text { Si le phénomène multimodal se prolonge sur plusieurs tours. On indique parfois le } \\
\text { numéro de la ligne correspondante à la fin du phénomène (ex : -->45). }\end{array}$ \\
\hline$\#$ & $\begin{array}{l}\text { Les images illustrant un phénomène multimodal sont notées par ce symbole qui est } \\
\text { temporellement aligné au verbal. }\end{array}$ \\
\hline
\end{tabular}

\section{NOTES}

1. Voir la description de cette relève dans la section suivante.

2. ELAN Linguistic Annotator, (C Max Planck Institute for Psycholinguistics, https:// tla.mpi.nl/tools/tla-tools/elan/

3. Computerized Language Analysis, (c) Leonid Spektor at Carnegie Mellon University, https://talkbank.org/

4. En analyse conversationnelle, de nombreux travaux ont été menés sur le rôle et les fonctions des regards dans la co-construction de l'interaction. Nous renvoyons entre autres à Goodwin (1980), Psathas (1990), Haddington (2006), Sidnell (2006), Rossano (2013), Streeck (2014) et Heath (2018).

\section{RÉSUMÉS}

La relève infirmière, liant des équipes de soins en psychiatrie, garante de la continuité des soins, est riche d'une interaction singulière et variée, et ne se limite pas à la simple transmission d'informations, mais tend à co-construire une réflexion d'équipe tournée vers le patient. L'approche interactionnelle (Grosjean \& Lacoste, 1999) et multimodale proposée ici vient mettre en lumière des compétences spécifiques de l'exercice infirmier au quotidien. À l'aide d'une captation audio-vidéo réalisée in situ, et dans une approche théorique s'inscrivant dans celle de l'analyse conversationnelle (Sacks et al., 1974), nous avons pu voir lors des réunions de relève, que l'actualité clinique du patient, autrement dit le «faire savoir», n'est pas la seule préoccupation des soignants. Une dimension didactique : le « faire apprendre » apparaît de façon assez fréquente, en rapport avec l'exercice infirmier en tant que tel, et non plus directement avec les patients. Le projet CIPSY permet la mise en avant d'une richesse «technique » de l'infirmier à travers des compétences spécifiques. Les analyses futures de ce corpus complexe constitué viendront illustrer la richesse du travail quotidien des infirmiers, l'analyse conversationnelle trouvant ici un terrain d'exploration nouveau et contributif à la qualité des soins.

The transmission of information concerning a patient at the hospital is governed on the one hand by the computerized patient's file, and on the other hand by the nurses' and/or multiprofessional's handovers. As a real challenge for the quality of healthcare, these oral and written transmissions are a very specific concern of healthcare institutions. Psychiatry, marked 
by orality (Barett, 1997), differs from the world of somatic care by this specificity. Nursing practice primarily uses relational care and uses a thought activity in the service of collective intelligence (Merkling, 2007). The CIPSY study (PSYchiatric Nurses Interaction Skills) conducted in collaboration between the Vinatier Hospital and the ICAR laboratory (CNRS, Lyon 2 University, ENS of Lyon) has the main objective of determining the language repertoire of the two production formats (oral and written) during nurses' handovers and written transmissions, using qualitative and quantitative analyses.

In this article, we propose to conduct an interactional and multimodal analysis of oral nurses' handovers in psychiatric institutions, which has emerged as a possible place to "know" and "learn" among health professionals. Although the main objective of a nurses' handover is to transmit and share the up-to-date information on the different patients present in the unit, we have been able to identify exchanges during which participants can "complement professional knowledge" or "help the partner see more clearly" (Grosjean \&Lacoste, 1999: 112). Indeed, as the authors have shown, "the role of interaction is not only to convey one way, it is to answer the questions and solicitations of others and integrate their reactions, it is to build a discourse thanks to the collaboration of many" (Grosjean \& Lacoste, ibid.).

Our analysis is based on four excerpts from the CIPSY project. The study includes multi-view audiovisual recordings of the three handovers on five consecutive Mondays of the same psychiatric unit: one at 6:30 a.m., one at 2:00 p.m. and one at 9:45 p.m. Added to these is the week's medical meeting, also known as the "weekly staff" which takes place at 9:00 a.m. The choice was only on Mondays because, for an adult psychiatry service and as the first day of the week, these are particularly rich handovers, since they are abundant of clinical events that happened during the weekend. The special feature of this day is also the weekly staff which exceptionally brings together nurses and nursing assistants in the morning, one or two psychiatrists, the psychologist, the health manager, the occupational therapist, the assistant students (nurses and externals). This original audiovisual corpus, with a total duration of 14 hours 30 minutes, depicts 38 health professionals, and concerns 40 patients put on the agenda of these meetings. Oral data are transcribed and coded in the ELAN software, written data (observations extracted from the patient record) are imported and encoded in the CLAN software. The coding grid of oral and written data is common to facilitate their comparison. It has been co-constructed with the recorded participants, and experts about care in order to stick to the problems of the service and to confront it with the knowledge of nursing experts.

The methodology of fieldwork, corpus collection and analysis in which the CIPSY project takes part is developed in interactional linguistics and conversational analysis. This methodology aims to capture audio and video data in order to make available, and therefore analysable, linguistic, multimodal and situational details (gazes, gestures, movements, actions, objects, bodily framework) relevant to recorded interaction (Groupe ICOR, 2006). It also allows us to observe precisely the evolution in time and space of activity and social interactions that co-construct between participants.

In this article, we propose to question the pre-established organization of nurses' handovers in a psychiatric unit and to identify moments that modify the planned interactional sequence of a meeting to bring a more didactic aspect to the current conversation. Indeed, the handovers follow a similar process. A particular nurse designates themself or is designated to list the clinical updated information of each patient, according to an order established by the room numbers. We will also use the concept of "super speaker" to define this central role in the succession. Other participants, on the other hand, can write down on a handover sheet what is said by the nurse in charge of the meeting. However, in four excerpts from the corpus, the didactic dimension is more present in the discourse of professionals and temporarily suspends the expected progression of the current transmission. We have identified two sequences with different didactic dimensions: the first is the repair of a multimodal transition between two patients (extracts 1 and 2), the 
second is the sharing of professional knowledge to colleagues (extracts 3 and 4). In an interactional and multimodal approach, we will describe the resources mobilized by the participants to support their discourse in these specific sequences. In particular, we will analyze the co-participants' management of an error in the transition from one patient to another, the suspension by a co-participant of the transition to another patient to finish taking notes on his sheet, the adjustment of the nurse in charge of the handoff to this suspension, or the emergence of exchanges of shared knowledge about the profession and the possible impact of these moments of regulation or shared knowledge on the effectiveness of the succession.

The results of these fine analyses highlight specific skills of daily nursing exercise. Indeed, we have seen that, during succession meetings, the patient's clinical news, in other words "knowing" it, is not the only concern of caregivers. While there is a strong tendency to describe only the psychological and somatic state of the patient, a didactic dimension appears quite frequently, in relation to nurses' work and skills, and no longer directly with patients' information. From a multimodal point of view, the results of the analyses show in particular that these more didactic moments are detectable by specific postures. In the "knowing" sequences, the note taking on their sheet by caregivers is a central activity and can modify the progression of transmissions, while in the didactic phases of "learning", the gazes are oriented more to the speaker who transmits knowledge and skills, stopping the writing activity. Future analyses of these successions will illustrate the richness of nurses' daily work. The conversational analysis here finds a new and contributing field of exploration to the quality of care.

\section{INDEX}

Mots-clés : relève, infirmière, interaction multimodale, faire savoir, faire apprendre

Keywords : handover, nurse, multimodal interaction, informing, upskilling

\section{AUTEURS}

\section{ISABEL COLÓN DE CARVAJAL}

ENS de Lyon, Laboratoire ICAR (UMR 5191)

isabelle.colondecarvajal@ens-lyon.fr

\section{LOUIS MARITAUD}

Université Lyon 2, Laboratoire ICAR (UMR 5191)

louis.maritaud@ens-lyon.fr

\section{BENOIT CHALANCON}

Centre hospitalier le Vinatier, Laboratoire ICAR (UMR 5191)

benoit.chalancon@ch-le-vinatier.fr

\section{JUSTINE LASCAR}

CNRS, Laboratoire ICAR (UMR 5191)

justine.lascar@ens-lyon.fr 